\title{
Open circuit voltage and efficiency in ternary organic photovoltaic blends
}

\author{
Nikolaos Felekidis, E. Wang and Martijn Kemerink
}

\section{Linköping University Post Print}

\section{Tweet}

N.B.: When citing this work, cite the original article.

Original Publication:

Nikolaos Felekidis, E. Wang and Martijn Kemerink, Open circuit voltage and efficiency in ternary organic photovoltaic blends, 2016, Energy \& Environmental Science, (9), 1, 257-266. http://dx.doi.org/10.1039/c5ee03095a

Copyright: Royal Society of Chemistry

http://www.rsc.org/

Postprint available at: Linköping University Electronic Press

http://urn.kb.se/resolve?urn=urn:nbn:se:liu:diva-124474 


\section{Journal Name}

\section{ARTICLE}

Received 00th January 20xx,

Accepted 00th January 20xx

DOI: $10.1039 / \times 0 \times x 00000 x$

www.rsc.org/

\section{Open circuit voltage and efficiency in ternary organic photovoltaic blends}

\author{
N. Felekidis, ${ }^{a}$ E. Wang*b and M. Kemerink*a,c
}

Organic bulk heterojunction solar cells based on ternary blends of two donor absorbers and one acceptor are investigated by experiments and modeling. The commonly observed continuous tunability of the open circuit voltage $\boldsymbol{V}_{\boldsymbol{O C}}$ with donor1:donor2 ratio can quantitatively be explained as quasi-Fermi level splitting due to photocreated charges filling a joint density of states that is broadened by Gaussian disorder. On this basis, a predictive model for the power conversion efficiency that accounts for the composition-dependent absorption and the shape of the current-voltage characteristic is developed. When all other parameters, most notably the fill factor, are constant, we find that for state-of-the-art absorbers, having a broad and strong absorption spectrum, ternary blends offer no advantage over binary ones. For absorbers with a more narrow absorption spectrum ternary blends of donors with complementary absorption spectra, offer modest improvements over binary ones. In contrast, when, upon blending, transport and/or recombination kinetics are improved, leading to an increased fill factor, ternaries may offer significant advantages over binaries.

\section{Broader context}

Solar cells based on blends of organic semiconductors almost routinely reach power conversion efficiencies (PCE) in the $10 \%$ range. One of the strategies that has been proposed to push this limit further upward is the use of so-called ternary blends in which two instead of one absorbing donor material is blended with the electron acceptor. Systematic improvement of PCE by ternary blending has been reported. Despite the experimental success, the current understanding of both the PCE enhancement and the underlying puzzling tunability of the open circuit voltage Voc is limited and essentially qualitative. In this contribution we develop and validate a quantitative and predictive framework for both the Voc and PCE of ternary organic solar cells, which allows us to propose design rules. In contrast to current wisdom, we demonstrate that PCE enhancement in ternary blends hardly results from optimizing the overlap with the solar spectrum but predominantly from synergetic improvement of the charge transport and recombination kinetics. The model can be easily extended to include more detail and will allow other researchers to upfront evaluate the photovoltaic potential of 'any' ternary material combination.

\footnotetext{
a. Complex Materials and Devices, Department of Physics, Chemistry and Biology (IFM), Linköping University, SE-581 83 Linköping, Sweden

b. Department of Chemistry and Chemical Engineering, Chalmers University of

Technology, SE-412 96 Göteborg, Sweden

Department of Applied Physics, Eindhoven University of Technology, PO Box 513, NL-5600 MB Eindhoven, The Netherlands

+ Footnotes relating to the title and/or authors should appear here.

Electronic Supplementary Information (ESI) available: [details of any supplementary information available should be included here. See DOI: 10.1039/x0xx00000x
} 


\section{Introduction}

Despite the very promising evolution of bulk heterojunction organic photovoltaics (BHJ-OPV), leading to single junction efficiencies currently exceeding $10 \% \mathrm{PCE}, 1,2$ incomplete absorption of the solar spectrum remains a limiting factor for the power conversion efficiency (PCE). Ternary BHJ, comprising two donors with complementary absorption spectra, promise to increase the generation of charge carriers as a result of increased sunlight absorption. ${ }^{3}$ However, the introduction of a second, more red-absorbing donor with a shallower HOMO is intuitively expected to pin the open circuit voltage $\left(V_{O C}\right)$ to the lower of the two values corresponding to the binary blends, thus limiting the PCE. Surprisingly enough, experiments show that absolute pinning does not take place. Instead, the opencircuit voltage in ternary OPV was shown to be continuously tunable with donor composition, ${ }^{4-7}$ allowing improvement of the PCE when combined with properly, i.e. complementary chosen donor absorption spectra. Likewise, addition of a second acceptor with a different LUMO level leads to a similar tunability of $V_{O C}{ }^{8,9}$

The reason for the continuously tunable Voc remains somewhat in the dark. Recently, Yang et al. proposed a 'parallel junction' model to phenomenologically describe this behavior. ${ }^{10}$ On the same basis Savoie et al. proposed an empirical expression for Voc as a current-weighted average of the two sub-systems and used this to estimate a maximal theoretical PCE (of 13\%) for varying donor bandgaps. ${ }^{11}$ Apart from lacking a formal basis, there are two problems with the parallel junction picture. First, it relies on a specific morphology in the active layer that allows different regions to act as independent binary cells that are electrically decoupled. Even if a fortunate morphology would lead to the presence of two (hole-) percolating networks that are electrically isolated from each other, the presence of metal contacts does unavoidably enforce equal quasi-Fermi levels in the subcells. The subcells cannot be treated independently. This problem becomes evident when considering the equivalent circuit of two parallel photodiodes. When biased beyond the lowest Voc, i.e. in the regime where the high-Voc subcell is supposed to boost Voc, the low-Voc subcell is in fact biased in its forward direction and will short the system. Alternatively, Street proposed a model for the formation of organic alloys in ternary blends based on two fullerene acceptors and a single donor. ${ }^{12}$ For this model to apply the charge wavefunction must be delocalized over many sites; for example for a 1:9 acceptor ratio, delocalization over more than 10 acceptor units is required to 'feel' on average at least one instance of each acceptor type. Whereas this may be conceivable for wellpacked fullerenes, this intuitively seems rather unlikely for mixtures of heterogeneous polymers. Nevertheless, Khlyabich et al. recently argued for such an alloying effect in polymers, although no explicit calculations of Voc were presented. ${ }^{4}$

Hence for a quantitative assessment of the (im)possibilities of ternary bulk heterojunction solar cells there is need for a somewhat more rigorous model. Here, we implement a simple physical model based on state filling in a disordered Gaussian density of states. By first assuming a constant occupation the experimentally observed gradual dependence of Voc on Donor1:Donor2 (D1:D2) ratio is explained. The assumption of constant occupation for all different polymer fractions is then expanded to include absorption and transport allowing a quantitative analysis of the achievable short circuit current $\left(j_{S C}\right)$ and power conversion efficiency in ternary blends. In agreement with experiments it is predicted that for materials with a wide and strong absorption spectrum no improvement can be expected when going from binary devices to ternary when all other parameters, like the fill factor, are unaffected by the blending. On the other hand a moderate improvement is expected for ternary over binary blends for more narrowspectrum absorbers. In contrast, we demonstrate that when the transport and/or recombination dynamics are simultaneously improved, ternary blends may offer significant advantages over binaries.

In the present work we focus on Donor1:Donor2: Acceptor BHJ OPV but the concept should be equally applicable to Donor: Acceptor1: Acceptor2 devices. It is unimportant if the constituent donor(s) and acceptor(s) are polymers, oligomers or small molecules. Moreover, for transparency we shall refer to the central energy of the disorder-broadened HOMO and LUMO levels as 'the' HOMO or LUMO energy, which differs from what is probed by common electrochemical methods like cyclic voltammetry. Also the optical bandgap, when taken to be the absorption onset, is substantially smaller than the HOMOLUMO gap due to (a) broadening and (b) excitonic effects. Because of the large uncertainties associated with both the absorption onset and the exciton binding energy we refrain from attempts to convert HOMO-LUMO gaps to optical gaps. This has no effect on the conclusions of this work.

\section{Results}

\section{Open circuit voltage of ternary blends}

In this section we will demonstrate why Voc does not pin to the HOMO of the shallower donor (Voc2 in Figure 1). In order to study the connection between the donor polymer composition and Voc different ternary BHJ OPV were fabricated by mixing the acceptor $\mathrm{PC}_{71} \mathrm{BM}$ with pairs of the donor polymers TQp6, TQm6 and TQm 12 in compositional steps of $10 \%$. The polymers were synthesized as indicated in Reference 13 and their molecular structures and full names can be found in Figure S1 in the Supporting Information (SI). These polymers were chosen for their similar structure leading to a good miscibility. Atomic force microscopy images were consistent with a good miscibility. We note that a poor miscibility of the two donors need not lead to different behavior. This is due to the presence of metal contacts that, as explained above, cause an equilibration of the Fermi levels throughout the device. However, for donor-donor demixing not to cause problems, it is essential that both donors (continue to) mix well with the acceptor, which leads to a system consisting of spatially separated mostly binary regions. Also the phase separation between the Donor1- and Donor2-rich phases should not cause additional morphological problems like overly rough or 
discontinuous films. The relative HOMO level energies of our donor polymers give rise to ternary systems with either a relatively small or a relatively large difference in Voc between the binary extremes. For all devices the total Donor:Acceptor ratio was kept constant at 1:1. For the given D1:D2 ratio the individual D:A ratio is not optimized as in this work transparency of results is favored over highest performance. Further details about device fabrication are given in the experimental section. The open circuit voltages under 1 Sun illumination vs. composition are shown in Figure 2.

The open circuit voltage of ternary systems based on TQm6:TQm12 (black symbols in Figure 2) and TQp6:TQm12 (blue symbols) show smooth and near-monotonous composition dependencies. The two outlying Voc data points of the TQm6:TQm12 at 0.5 and 0.6 polymer ratios were investigated by AFM but no systematic (deviations in) phase separation was observed (Figure $\mathrm{S} 2$ in $\mathrm{SI}$ ); neither did any of the other performance indicators indicate specific problems for these two devices. Irrespective of the particular functional shape, all ternary systems are consistent with previous reports that Voc is continuously tunable via the D1:D2 ratio. Moreover, they strongly suggest an increasing bowing of the trend line with increasing difference between the Voc's of the binary extremes. This is better visible in the inset to Figure 2 where the normalized Voc shifts are plotted.

In order to rationalize these observations, we apply a simple state filling model where the disordered Gaussian density of states for the two donor polymers and the acceptor are defined according to

$$
G(E)=\frac{1}{\sigma \sqrt{2 \pi}} \exp \left(-\frac{\left(E-E_{\text {HOMO/LUMO }}\right)^{2}}{2 \sigma^{2}}\right)
$$

$E_{\text {HOMO }}$ and $E_{L U M O}$ are the donor and acceptor energy levels and centers of the Gaussian DOS. The parameter $\sigma$ defines the broadening of the DOS. The independent variable $E$ is the energy. In the present simulation the relevant energy levels are the HOMO levels of the two donors and the LUMO level of the acceptor. The effective hole DOS, $G_{\text {eff }}$ is calculated according to

$$
G_{\text {eff }}=f_{D 1} \cdot G_{D 1}+\left(1-f_{D 1}\right) \cdot G_{D 2}
$$

where $f_{D 1}$ is the composition ratio of $\mathrm{D} 1$ to the total donor concentration and $G_{D 1}, G_{D 2}$ are the Gaussian DOS of the two polymers (Equation 1). For electrons, that reside on the single acceptor, $G_{e f f}=G$. The key assumption at this point is that the electron and hole populations in the entire $\mathrm{BHJ}$ are in equilibrium, i.e. there are no lateral variations in the quasi Fermi levels $E_{F}$. Hence, the electron quasi Fermi level $E_{F, \text { el }}$ can be calculated from the condition that

$$
\frac{n}{N_{0}}=\int_{-\infty}^{\infty} f_{F D}\left(E-E_{F, e l}\right) \cdot G_{e f f}(E) d E
$$

where $n / N_{0}$ is the electron occupation with $N_{0}$ the total site density and $f_{F D}\left(E-E_{F, e l}\right)$ the Fermi-Dirac distribution

$$
f_{F D}\left(E-E_{F, e l}\right)=\frac{1}{1+\exp \left(\frac{E-E_{F, e l}}{k_{B} T}\right)}
$$

The temperature is set at $300 \mathrm{~K}$ and $k_{B}$ is Boltzmann's constant. For holes similar expressions are used to obtain $E_{F, \text { hole }}$ from $p / P_{0} . V_{O C}$ is then obtained as the difference between the electron and hole quasi Fermi levels $E_{F, e l}$ and $E_{F, h o l e}$ :

$$
V_{O C}=E_{F, e l}-E_{F, \text { hole }}
$$

The results of the aforementioned physical model are depicted in Figure 2 as solid lines. In these simulations the (hole) occupation is an effective parameter, accounting for light intensity and absorption, transport, recombination etc., that is kept constant for all compositions. The main trends of the experiments, i.e. a continuous transition between the binary extremes at $x=0$ and $x=1$ with a bending that increases with increasing $\triangle \mathrm{HOMO}$, are well reproduced. This behavior is explained in Figure 3 where the effect of the donor HOMO level offset $\triangle \mathrm{HOMO}$ on the composition dependence of the quasi Fermi level for holes, and hence on $V_{O C}$ is demonstrated. The left panel of the figure shows a pure Donor1 (D1): acceptor (A) composition resulting in a deep Fermi level for the holes. When a small fraction of a second donor (D2) with a less deep HOMO level is added, two extreme scenarios can be discerned. When the HOMO of the second donor is far from that of D1, it will effectively pin the hole Fermi level since (a) the occupation is always much less than the fraction of D2 and (b) the DOS of D1 is negligible at the Fermi energy: all holes sit on D2 sites. When the HOMO of the second donor is close to that of D1, condition (b) above is no longer met, and the Fermi level is determined by the relative weights of the D1 and D2 DOS at the Fermi energy, giving rise to a more gradual shift of $E_{F, \text { hole }}$ with D1:D2 ratio.

As a further confirmation we used the same model to successfully describe experimental Voc data for three optimized D1:D2:A ternary systems reported in literature. ${ }^{4,5}$ The fitting results and used parameter values are shown in the SI as Figures S5,S6,S7 and Tables T2,T3,T4 respectively. These fittings confirm the conclusion from Figures 2 and 3 above, that larger differences in Voc between the binary extremes lead to more pronounced curvature of the Voc vs. composition curve. Importantly, it does so without having to invoke any structural or alloying effects. . $^{4}$

Having established a simple, but physically consistent model to describe the composition dependence of Voc in ternary blends, the question arises if the 'tunability' of Voc indeed allows ternary OPV to outperform binaries. Up to now simulations are based on the assumption that occupation is constant for all compositions, a fact that does not allow addressing the key (supposed) advantage of ternaries, a better overlap with the solar spectrum, leading to higher $j_{S C}$ and PCE. In the next section we will therefore extend our model to include the composition dependent absorption.

\section{Power conversion efficiency of ternary blends}

The need of quantifying the occupation of the density of states, i.e. knowing the number of charge carriers in the device, was solved by implementing an optical model that takes into account the net absorption of the constituent absorbers, here 
assumed to be the donors. The respective absorption lengths $L_{D 1,2}$ as a function of wavelength are given by

$$
\begin{gathered}
L_{D 1}(\lambda)=\frac{L_{D 10}}{G_{D 1}(\lambda) \cdot f_{D 1}} \\
L_{D 2}(\lambda)=\frac{L_{D 20}}{G_{D 2}(\lambda) \cdot\left(1-f_{D 1}\right)}
\end{gathered}
$$

where $L_{D 10}, L_{D 20}$ is the absorption length (or attenuation length) of the pure material and $f_{D 1,2}=D 1 /([D 1]+[D 2])$ the Donor1 fraction. $G_{D 1,2}(\lambda)$ is the absorption profile that is assumed to be Gaussian -for organic semiconductors this choice is more realistic than a step-like absorption onset as used in e.g. the Shockley-Queisser analysis: ${ }^{14}$

$$
G_{D 1,2}(\lambda)=\exp \left(-4 \log 2\left(\frac{\lambda-E_{g 1,2}}{W_{D 1,2}}\right)^{2}\right)
$$

with $E_{g 1,2}$ and $W_{D 1,2}$ the maximum and full width at half maximum (FWHM) -both in $\mathrm{nm}$ - of the absorption. The absorption maximum corresponds to the HOMO-LUMO gap as described in the previous section and Figure 1. $E_{g 1,2}$ and $W_{D 1,2}$ are chosen to fit the real absorption spectrum of the donors under study (Figure S8 in $\mathrm{SI}$ ). Although we will not pursue this here, we note in passing that Eq. 7 can readily be modified to account for additional absorption maxima or the absorption of sensitizers. ${ }^{3,10}$

The effective absorption length $L_{e f f}$ for the blend is given by

$$
\frac{1}{L_{e f f}(\lambda)}=\frac{1}{L_{D 1}(\lambda)}+\frac{1}{L_{D 2}(\lambda)}
$$

For our semi-transparent devices of device thickness $L_{\text {device }}$ the absorption is approximated as

$$
A(\lambda)=1-\exp \left(-\frac{L_{\text {device }}}{L_{\text {eff }}(\lambda)}\right)
$$

Using the absorption spectrum Equation 9 the flux of photogenerated holes and electrons is calculated by integrating the product of the solar spectrum and the absorption spectrum as

$$
\dot{n}=\dot{p}=\frac{1}{L_{\text {device }}} \int \frac{A M 1.5(\lambda) \cdot I Q E \cdot A(\lambda)}{E_{p h}(\lambda)} d \lambda
$$

where IQE is the internal quantum efficiency that was set at 0.85 defining a moderate device performance. AM1.5 is the solar spectrum and $E_{p h}$ is the photon energy in eV needed for dimensional reasons. Again, we note that Eq. 10 can be readily extended to include more advanced absorption models that take into account e.g. reflection losses of cavity effects. For the present purposes, focusing on relative differences and semitransparent cells, there is little need to do so.

In order make the connection of the above to experiments as straightforward and transparent as possible, we adopt a phenomenological transport model. This avoids having to explicitly address the complicated charge transport in the blend, including morphological effects, (reduced) bimolecular and trap-assisted recombination, density and field dependent mobilities etc. The free carrier concentration is approximated by multiplying the carrier flux $\dot{n}$ or $\dot{p}$ with the respective lifetime $\tau_{e l}$ or $\tau_{\text {hole }}$ :

$$
\begin{gathered}
n=\dot{n} \cdot \tau_{e l} \\
p=\dot{p} \cdot \tau_{\text {hole }}
\end{gathered}
$$

Although the lifetimes of electrons and holes can be expected to be different, we found that using different values led to an under-determinedness in our model. When running simulations in which the electron and hole lifetimes were varied between 1 and $100 \mu \mathrm{s}$, both concertedly and independently, we found only minor quantitative differences. The conclusions from the following sections are therefore independent of charge carrier lifetimes. Hence $\tau_{e l}$ and $\tau_{\text {hole }}$ are taken equal and set at $10 \mu \mathrm{s}$. This assumption corresponds to the optimal situation of balanced charge transport, which also minimizes space charge effects as it leads to $n=p$.

$V_{O C}$ is calculated from the occupation and quasi Fermi levels as described earlier in Equations 3-5. Finally, the short circuit current $j_{S C}$ and the power conversion efficiency PCE are calculated as

$$
\begin{gathered}
j_{S C}=q \cdot \dot{p} \cdot L_{\text {device }} \\
P_{\text {out }}=F F(\ldots) \cdot V_{\text {OC }} \cdot j_{S C} \\
P C E=P_{\text {out }} / P_{\text {in }}
\end{gathered}
$$

where $F F(\ldots)$ is the fill factor that in principle is a complicated and essentially unknown function of many parameters. $P_{\text {in }}$ is the input solar power $\sim 1 \mathrm{~kW} / \mathrm{m}^{2}$ defined as the integral of the AM1.5 spectrum over the wavelength. As we primarily are interested in differences in PCE as function of HOMO and LUMO energies, the fill factor will be taken constant. The dependency of the PCE on FF will be explicitly addressed in the final section of this paper.

As a first test, by implementing the optical model using the parameter values in Table T1 in the SI, the calculated Voc, $j_{S C}$ and PCE closely fit the trends in the experimental data of the TQm6:TQm12:PC ${ }_{71} \mathrm{BM}$ system as seen in Figure 4 . The simulation results show the expected increasing current with decreasing open circuit voltage due to an increased absorption. The fill factor for this specific blend was measured to be fairly constant at 0.45 and this value was used in the simulations. As mentioned above, the three donor polymers were selected to give, in combination with PCBM, two ternary systems with a weak respectively a strong composition dependence of Voc, see Figure 2. At the same time, the rather similar absorption spectra lead to an absence of clear trends in $j_{s c}$ and PCE for the systems where TQp6 is mixed with TQm6 and TQm12 polymers, that can, however, still be surprisingly accurately reproduced given the simplicity of the model presented above (see SI Figures S9, S10 and Tables T6, T7).

\section{Selecting optimal donor materials and compositions}

In this final section the performance limits of ternary OPV will be assessed. The goal is to establish an estimate for the maximum improvement ternary devices may have over the 
corresponding binary devices. For the following calculations we keep all parameters constant, and equal to experimentally realistic values, except for the LUMO or the HOMO levels of the donors. As a consequence, these calculations focus on ternary systems with complementary absorption spectra, in contrast to the materials used in the experiments discussed above. Since we are interested in the maximum achievable PCE, for each specific pair of donors, with given HOMO and LUMO levels, the ratio of the concentration of the first donor to the total donor concentration $D 1 /(D 1+D 2)$ that gives rise to the highest PCE is calculated. An example of a typical PCE vs. D1/(D1+D2) curve is given in the $\mathrm{SI}$, Figure $\mathrm{S} 11$.

Case 1 - LUMO variation. This case is inspired by the work of Yang et al., ${ }^{15}$ who investigated the performance of ternary OPV for a range of polymer donors with nearly identical HOMO levels and varying LUMO level. This system is unlikely to lead to an optimal ternary device in absolute sense for two reasons. First, by having a fixed HOMO level it cannot profit from voltage gains associated with adding a more 'blue' absorber, cf. Figures 1, 2. Second, varying the LUMO level implies that mostly nonoptimal LUMO offsets between donor(s) and acceptor, causing energy losses upon charge transfer, will be evaluated. The results of this type of ternary system are nevertheless very instructive.

In these simulations the LUMO levels for both donor 1 and 2 are varied between $-2.5 \mathrm{eV}$ and $-4.1 \mathrm{eV}$ while the HOMO level is fixed at $-5.4 \mathrm{eV}$. The acceptor LUMO level is the one for $\mathrm{PC}_{71} \mathrm{BM}$, set at $-4.1 \mathrm{eV}$. Where possible we used parameters that correspond to Reference ${ }^{15}$ (see SI Table T8). The results of the simulations are shown in Figure 5, with the left panel showing the optimal D1 fraction for the given LUMO level combination and the right panel the corresponding PCE, i.e. at the optimal D1 fraction.

In agreement with Reference ${ }^{15}$, for most D1-D2 combinations the optimal composition is a ternary one, i.e. in Figure 5a bright yellow (pure D1) and blue (pure D2) regions are relatively rare and do not come near the overall optimal PCE of $8.75 \%$ that is found for a ternary system with $D 1: D 2 \approx 1: 1$. The latter is rather close to the value of $8.5 \%$ that is reported by Yang et al. for (PTB7:PBDTT-SeDPP = 1:1):PC ${ }_{71}$ BM. The donor LUMO levels for which the optimal PCE is found in the simulations, $\sim-3.25 \mathrm{eV}$ and $\sim-3.85 \mathrm{eV}$ are very consistent with the $-3.31 \mathrm{eV}$ and $-3.70 \mathrm{eV}$ of PTB7 and PBDTT-SeDPP, respectively.

The reason why, in the case of a fixed and common donor HOMO level, ternary systems outperform binary ones is the following. The constant HOMO energy makes Voc essentially constant, so the only thing to be optimized is the overlap with the solar spectrum. For a given finite absorption width two donors are, in virtually all cases, better at that than a single one. For the used parameters, the results indicate that ternary devices are the better choice over binary and that they can increase the PCE by $\sim 33 \%$ as the best binary has a PCE of $2.5 \%$ whereas best ternary has $8.75 \%$. For comparison, Yang et al. find binary PCE between $6.2 \%$ and $7.2 \%$, again consistent with our model. It should, however, be stressed that the superiority of ternary OPV is not a general result; especially when not the donor HOMO but the donor LUMO is kept fixed. In this case higher performances can be achieved, leading to a dramatically changed picture.

Case 2 - HOMO variation. In this case the LUMO level of the donors is fixed at $-3.8 \mathrm{eV}$ creating a (near) optimal offset of 0.3 $\mathrm{eV}$ from the $\mathrm{PC}_{71} \mathrm{BM} L U M O$ which we fixed at $-4.1 \mathrm{eV}$. In contrast to the previous case with a fixed HOMO/variable LUMO, changing the HOMO affects both Voc and the overlap with the solar spectrum, i.e. $j_{S C}$ : a deep HOMO level increases Voc, but also the absorption bandgap which eventually reduces the current. On the other hand a more 'red' absorber maximizes overlap with the solar spectrum and $j_{S C}$, but the required shallow HOMO level will result in a smaller Voc.

An important technicality that needs to be addressed here is the sensitivity of the model results to the choice of parameters. First, all results presented in this work are robust to parameter variations in the sense that the qualitative conclusions remain unaffected. However, quantitative results do depend on parameter choices and especially on the shape of the absorption profile. For the calculation of Figure 5 above quantitative agreement with Reference 15 in Figure 5 is best when a nm-axis is used. The reason for that is likely the fact that the actual absorption profiles are rather symmetric on a $\mathrm{nm}$ axis. For the present calculation (variable HOMO) it turns out that evaluating Equation 7 on a nm-axis leads to results that are in conflict with experimental observations. Therefore in the following an eV-axis will be used; otherwise all parameters are kept as in Figure 5.

The results of Figure 6 indicate a highest achievable PCE of $\sim 13 \%$. Surprisingly, this is found for binary devices -in Figure $6 \mathrm{~b}$ the superiority of binary devices shows up as a yellow cross. Mapping this cross on panel a shows that the horizontal and vertical branches correspond to pure D1 and pure D2, respectively. The two small ternary regions, indicated by dashed ellipses, show that for certain polymer combinations optimized ternary systems can have an improved PCE over the corresponding binaries. At the same time, these specific ternaries do no not give an optimal overall PCE.

There are multiple reasons for the abrupt jumps in optimal composition in Figure 6a. The jump around the 'binary line' (dashed diagonal), reflects the fact that the overall-optimal device is binary: for $D 1,2$ combinations where neither donor is optimal, still the optimal D1:D2 ratio is the one where one has as much as possible from the donor that comes closest to the optimal donor, i.e. again a binary system. In other words, disadvantages in terms of reduced current or Voc outweigh any advantages of having a ternary system. The other jumps are associated with the presence of two maxima in the PCE vs. composition curve as explained at Figure S11 in the SI.

It is important that the maximum PCE is now predicted to be substantially larger than in the previous variable-LUMO case. The reason is that in the present case energy losses associated with electron transfer to the acceptor are much reduced. This is equivalent to the fact that increasing the bandgap in this 
(variable HOMO) case does, but in the other (variable LUMO) case does not lead to an increased Voc, while having the same effect on the short circuit current.

Comparing to the work of Scharber et al. on donor selection rules $^{16}$, where they predict a PCE of $10 \%$ for binary devices, the higher value of $13 \%$ that is found here can mostly be attributed to the used higher IQE of $85 \%$ (instead of $65 \%$ ). A close fitting of the present model to the one of Scharber et al. is included in Figure S12 using the parameters of table T9.

A remarkable observation in Figure 6 is the position of the optimal HOMO level at $-5.78 \mathrm{eV}$, corresponding to a bandgap of $\sim 2.0 \mathrm{eV}$. The difference with experimental findings where optimal HOMO energies around $-5.3 \mathrm{eV}$ are typically found ${ }^{1,2,15}$ is partially due to differences in the way the bandgap and HOMO, LUMO energies are defined. Here, central energies are used; the parameters in Figure 6 give in fact rise to an absorption onset at $\sim 1.5 \mathrm{eV}(\sim 830 \mathrm{~nm})$, in good agreement with the PTB7 absorption spectrum in Reference ${ }^{15}$ that we took as reference for the absorption width. At the same time, the present results indicate that substantial improvement in PCE can be obtained for a PTB7-like material with the same bandgap but downward-shifted HOMO and LUMO levels -PTB7 has a LUMO at $\sim-3.3 \mathrm{eV}$, giving rise to a large charge transfer energy loss. This suggestion is corroborated by the results of $\mathrm{He}$ et al. who show that PTB7-Th, having slightly deeper HOMO and LUMO levels, indeed gives improved performance. ${ }^{2}$

Using parameters that correspond to current state-of-the-art donor materials, ternary compositions show no improvement in PCE over binaries for the vast majority of energy level positions (Figure 6). The picture becomes different if a less optimal absorber is used. Setting the absorption FWHM from $0.8 \mathrm{eV}$ in Figure 6 to $0.5 \mathrm{eV}$ leads to a situation where the overall optimal PCE is produced by a ternary blend, see Figure 7. Note that this FWHM corresponds to Reference ${ }^{10}$ where, at the same time, rather optimistic absorption lengths of $10-20 \mathrm{~nm}$ were used in combination with a $200 \mathrm{~nm}$ device thickness. In Figure $7 \mathrm{~b}$ a clear cross shape, corresponding to near-optimal binaries is visible. As a general design rule we find that narrow but strong absorbers give rise to systems where ternary compositions offer improved performance over binary ones; broadening the absorption (cf. Figures 6,7 ) or weakening the absorption length shifts the balance to binary-dominated ones. This is further illustrated in Figure S13 of the SI. The rationalization of this rule consists of two parts. First, for strong absorbers the penalty for diluting the absorber that is closest to the optimal donor by adding a small fraction of another donor, is not so large as most light will still get absorbed. Second, for narrow absorbers the gain of adding another absorption band is relatively large, especially if the added absorber is a strong one.

An instructive intermediate situation arises when a material with a wide absorption band, as in Figure 6, is mixed with one with a narrow absorption, as in Figure 7. Intuitively one might expect the narrow band to complement the wide one. It does, but at the price of diluting the wide absorption material. The net result is a current and PCE loss with respect to the overall optimal binary. Alternatively, one can rationalize this result on basis of Figure 6 by realizing that adding a narrow absorber must in all cases be inferior to adding a wide one.

The above findings lead to the conclusion that in the case of realistic good absorbers with close to optimal energy levels the enhanced overlap with the solar spectrum cannot be expected to lead to significant improvement in performance of ternary blends of the D1:D1:A type. However, this conclusion depends critically on the assumption that all other parameters are independent of composition. In practice, especially the fill factor can depend significantly on composition, as e.g. in References $6,7,17$, leading to optimal ternary compositions. Where this is not the case, e.g. in References ${ }^{5,18}$, optimal PCE are found for close to binary compositions, in agreement with the conclusions from Figures 6 and 7 .

In order to illustrate the critical role the composition-dependent fill factor plays in achieving ternary systems that outperform their binary counterparts, the simulations of Figure 6 were reran with a fill factor that is a function of the D1 fraction $f_{D 1}$ :

$$
F F\left(f_{D 1}\right)=a f_{D 1}^{2}+\left(F F_{D 1}-F F_{D 2}-a\right) f_{D 1}+F F_{D 2}
$$

with $F F_{D 1,2}$ the fill factor at the binary extremes corresponding to donor 1 and 2 and $a$ a bowing constant. A positive (negative) bowing parameter $a$ reflects degraded (improved) charge transport and recombination kinetics with respect to a linear interpolation between the binary extremes and can e.g. result from morphological effects like changes in molecular packing and phase separation.

In the simulations of Figure 8 we used typical values of $F F_{D 1}=$ $0.65, F F_{D 2}=0.5$ and $a=-0.45$, corresponding to a maximum $\mathrm{FF}$ of 0.7 at $f_{D 1}=0.66$, as shown in Figure S14 in the SI. The calculation results now show that for virtually all HOMO level combinations, including the overall-optimal one, a ternary compound is preferred. Comparison of this result with that in Figure 6 allows two important conclusions. First, in case of ternary blending using state-of-the-art absorbers, minor or no improvements due to a better overlap with the solar spectrum can be expected (Figures 6,7), but 'secondary effects' that lead to an improved fill factor can give rise to significant improvements (Figure 8). This is in agreement with the experimentally observed correlation between optimal fill factor and optimal PCE. $6,7,17$ Second, in Figure 8 the overall optimal PCE is still located on the binary line, where both donors have the same HOMO and LUMO. This reiterates the superiority of a single optimal bandgap provided the absorption is sufficiently broad. Hence, the ideal ternary system consists of two heterogeneous donors, both having this optimal bandgap, and showing synergistic behavior in terms of FF, i.e. a negative $a$ in Eq. 13. Experimentally this may be hard to achieve and one may be forced away from the overall PCE optimum.

As for our own experimental system, that was selected to investigate $V_{O C}$, the virtual absence of spectral differences between the donors and the composition-independent fill factor preclude ternary compositions from having any advantages over binaries.

We shall finally draw an analogy between the present results and the 3-phase morphology encountered in binary OPV systems in which pure and aggregated donor and acceptor 
phases coexist with an amorphous mixed phase. ${ }^{19}$ Ignoring complications associated with the fact that charge transfer to the acceptor may be affected for better or worse, the mixed and pure donor phases can be considered as donor 1 and donor 2 phases, with slightly shifted energy levels due to the aggregation. From the discussion of Figures 6 and 7 it then follows that such a 3-phase morphology is sub-optimal in case of a donor with a broad absorption spectrum.

\section{Experimental}

Bulk heterojunction ternary (D1:D2:A with $(D 1+D 2): A=1: 1$ weight ratios) OPV where made from polymer-fullerene solutions of $25 \mathrm{~g} / \mathrm{L}$ concentration in 1,2-dichlorobenzene (ODCB) according to the following process. A $40 \mathrm{~nm}$ thin film of poly(3,4-ethylenedioxythiophene) polystyrene sulfonate (PEDOT:PSS) was spin coated on pre-cleaned ITO/glass substrates in air. After baking the active layer was spin coated in a glove box. A $0.6 / 90 \mathrm{~nm} \mathrm{LiF} / \mathrm{Al}$ top contact was evaporated on the active layer under high vacuum conditions. The device areas and thicknesses where measured to be $0.044-0.048 \mathrm{~cm}^{2}$ and $90-110 \mathrm{~nm}$ respectively. The $j V$-curves were measured under simulated AM 1.5 illumination. For all material combinations (TQm6:TQm12, TQp6:TQm6, TQp6:TQm12) device performance indicators $\left(j_{S C}, V_{O C}, \mathrm{PCE}\right)$ were measured and averaged on typically 3 devices on 2 different samples ( 6 devices in total), leading to relative errors that never exceeded $5 \%$.

\section{Conclusions}

We have investigated the device physics of organic bulk heterojunction solar cells based on ternary blends consisting of two absorbing donors (D1,2) and a single acceptor, focusing on the relation between the D1:D2 ratio and the open circuit voltage $V_{O C}$ and on the maximally attainable power conversion efficiency (PCE). We experimentally demonstrated that the shape of the $V_{O C}$ vs D1-fraction curve depends on the difference between the $V_{O C}$ of the binary extremes. Larger $V_{O C}$ differences give rise to a stronger curvature, i.e. a faster drop of $V_{O C}$ to the lowest binary value. This behavior is quantitatively reproduced by a simple physical model that is based on the notion that photocreated charges fill the joint density of states. By incorporating a simple absorption model that accounts for the composition dependent overlap with the solar spectrum we are able to make quantitative predictions of the PCE of ternary systems. The PCE model compares favorably to available literature data and indicates that for state-of-the-art absorbers, having a wide and strong absorption spectrum, ternary blends offer no advantages over optimized binaries when all other parameters, most notably the fill factor, are constant. When, in contrast, a composition-dependent fill factor is used, reflecting improved transport and/or recombination kinetics for ternary compositions, the latter gain a clear advantage over binaries. Hence, our results suggest that the advantage of ternary OPV systems should not be sought in improved overlap with the solar spectrum but in synergistic effects related to charge transport and recombination, leading to a higher fill factor. As compared to blending electro-optically inert materials like poly(styrene), which have been shown to improve the transport characteristics of small molecule OPV systems, ${ }^{20}$ the crucial advantage of blending in a second absorbing and transporting material is that the absorption can remain (mostly) uncompromised, avoiding the need for thicker layers.

\section{Acknowledgements}

EW acknowledges the Swedish Research Council for financial support.

\section{Notes and references}

1 Y. Liu, J. Zhao, Z. Li, C. Mu, W. Ma, H. Hu, K. Jiang, H. Lin, H. Ade, H. Yan, Nat. Commun. 2014, 5, DOI 10.1038/ncomms6293.

2 Z. He, B. Xiao, F. Liu, H. Wu, Y. Yang, S. Xiao, C. Wang, T. P. Russell, Y. Cao, Nat. Photonics 2015, advance online publication, DOI 10.1038/nphoton.2015.6.

3 T. Ameri, P. Khoram, J. Min, C. J. Brabec, Adv. Mater. 2013, 25 , 4245.

4 P. P. Khlyabich, Andrey E. Rudenko, Barry C. Thompson, and Yueh-Lin Loo, Adv. Funct. Mater. 2015, 25, 5557

5 P. P. Khlyabich, B. Burkhart, B. C. Thompson, J. Am. Chem. Soc 2012, 134, 9074

6 Y. Zhang, D. Deng, K. Lu, J. Zhang, B. Xia, Y. Zhao, J. Fang, Z. Wei, Adv. Mater. 2015, 27, 1071.

7 G. Williams, H. Aziz, Org. Electron. 2015, 17, 229.

8 P. Cheng, Y. Li, X. Zhan, Energy Environ. Sci. 2014, 7, 2005.

9 P. P. Khlyabich, B. Burkhart, B. C. Thompson, J. Am. Chem. Soc 2011, 133, 14534.

10 L. Yang, H. Zhou, S. C. Price, W. You, J. Am. Chem. Soc. 2012, 134,5432

11 B. M. Savoie, S. Dunaisky, T. J. Marks, M. A. Ratner, Adv. Energy Mater. 2015, 5, 1400891.

12 R. A. Street, D. Davies, P. P. Khlyabich, B. Burkhart, B. C. Thompson, J. Am. Chem. Soc. 2013, 135, 986.

13 E. Wang, J. Bergqvist, K. Vandewal, Z. Ma, L. Hou, A. Lundin, S. Himmelberger, A. Salleo, C. Müller, O. Inganäs, F. Zhang, M. R. Andersson, Adv. Energy Mater. 2013, 3, 806.

14 W. Shockley, H. J. Queisser, J. Appl. Phys. 1961, 32, 510.

15 Y. (Michael) Yang, W. Chen, L. Dou, W.-H. Chang, H.-S. Duan, B. Bob, G. Li, Y. Yang, Nat. Photonics 2015, 9, 190.

16 M. C. Scharber, D. Mühlbacher, M. Koppe, P. Denk, C. Waldauf, A. J. Heeger, C. J. Brabec, Adv. Mater. 2006, 18, 789

17 S.-J. Ko, W. Lee, H. Choi, B. Walker, S. Yum, S. Kim, T. J. Shin, H. Y. Woo, J. Y. Kim, Adv. Energy Mater. 2015, 5, 1401687.

18 L. Lu, T. Xu, W. Chen, E. S. Landry, L. Yu, Nat. Photonics 2014, $8,716$.

19 S. Shoaee, S. Subramaniyan, H. Xin, C. Keiderling, P. B Tuladhar, F. Jamieson, S. A. Jenekhe, J. R. Durrant, Adv. Funct Mater. 2013, 23, 3286.

20 Y. Huang, W. Wen, S. Mukherjee, H. Ade, E. J. Kramer, G. C. Bazan, Adv. Mater. 2014, 26, 4168. 


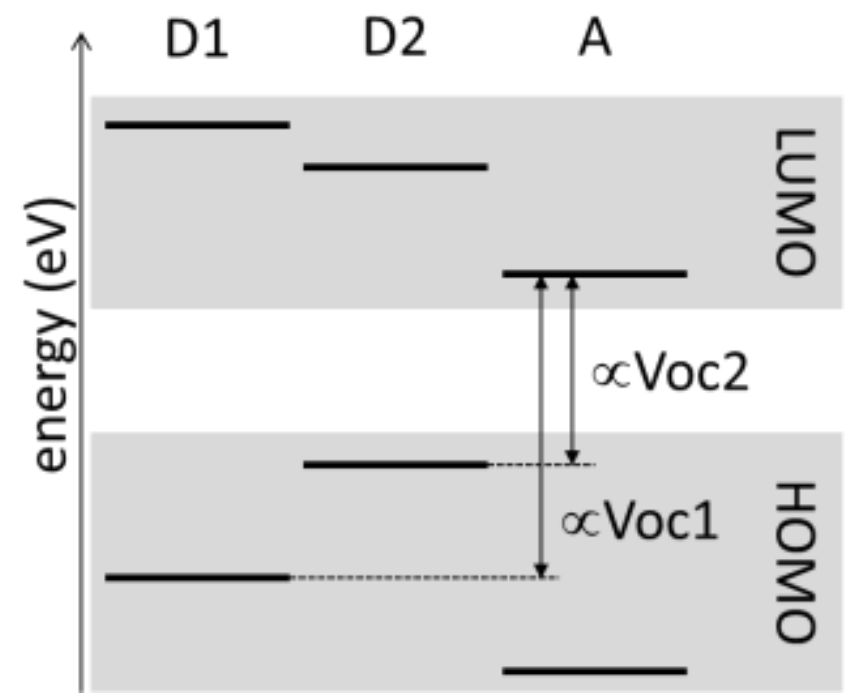

Fig. 1 Schematic band diagram depicting the offset of LUMO and HOMO levels for a donor pair D1, D2 and an acceptor A. Limiting binary Voc's are indicated as being proportional to the difference between the LUMO of the acceptor and the HOMO of the donors. The Voc of the D1:D2:A ternary blend is continuously tunable between Voc1 and Voc2.

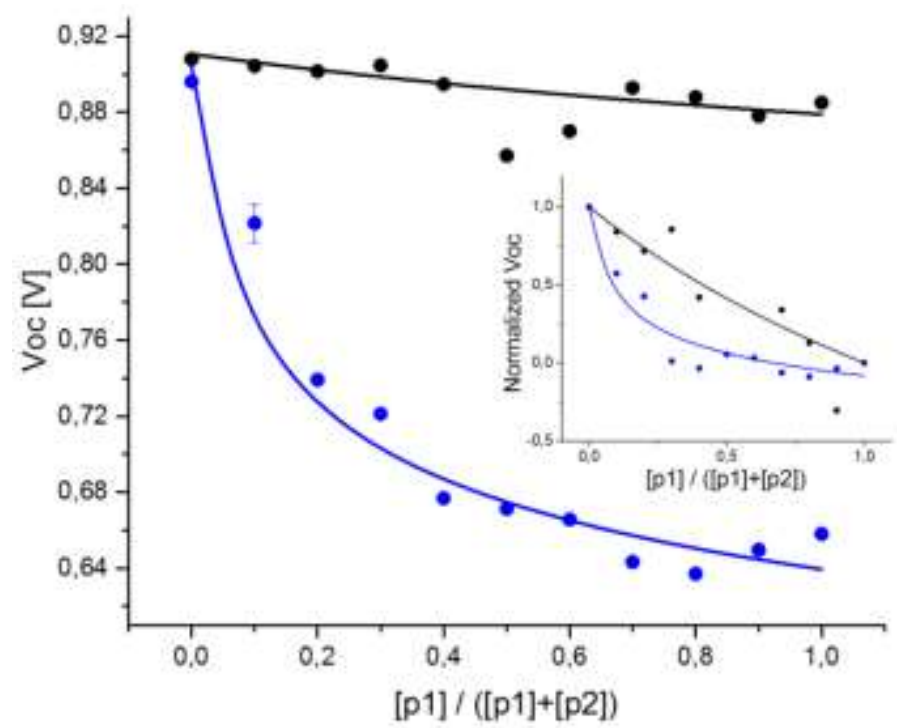

Fig. 2 Experimental (symbols) and simulation (lines) data of Voc vs composition for TQm6:TQm12:PC ${ }_{71} \mathrm{BM}$ (black) and TQp6:TQm12:PC ${ }_{71} \mathrm{BM}$ (blue) DDA ternary blends. The $\mathrm{x}$-axis is the ratio of the concentration of the first polymer to the total polymer concentration. The estimated standard deviation in Voc as determined from several devices on multiple substrates did not exceed $10 \mathrm{meV}$ (plotted error bar) for any of the Voc measurements. The parameter values used for the simulations are listed in Table T1 in the SI. The inset shows the same data normalized to the values at $x=0$ and $x=1$. 

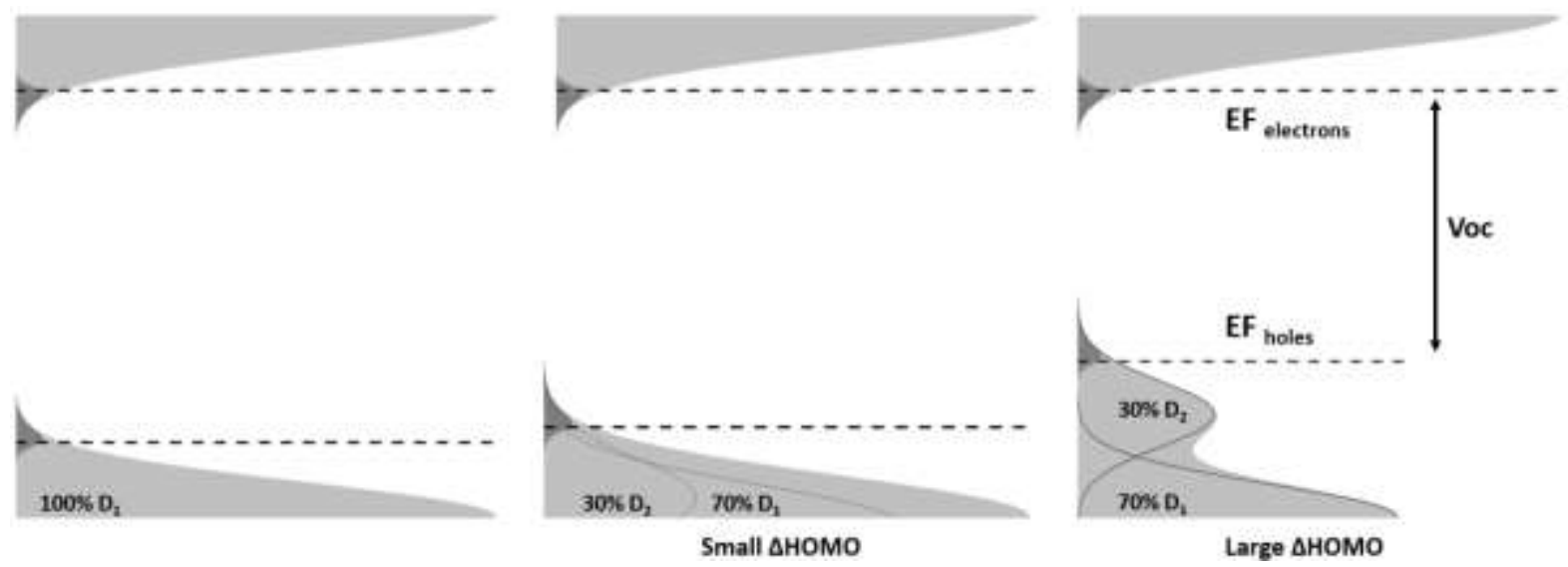

Fig. 3 Schematic representation of the calculated Voc as the difference between the electrons and holes quasi-Fermi level for a given occupation. Left: binary D1:A; middle, right: D1:D2 = 0.7:0.3 and small resp. large HOMO level difference. The DOS and DOOS of the donors and acceptor are represented by light and dark gray areas, respectively. The DOS contributions of D1 and $\mathrm{D} 2$ are shown by dark gray lines.
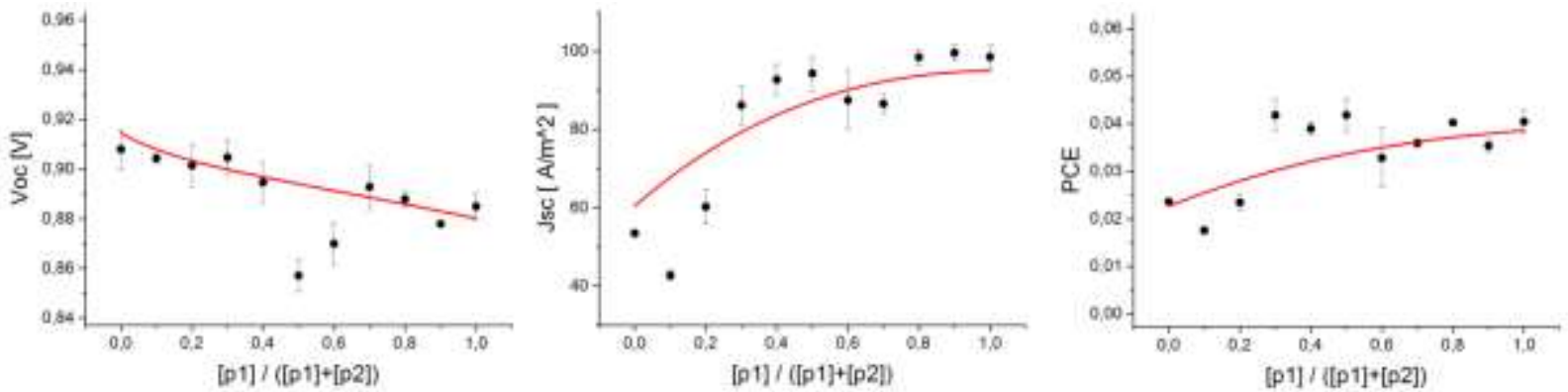

Fig. 4 Simulation (lines) and experimental (symbols) data for $\operatorname{Voc}(\mathrm{a}), j_{S C}$ (b) and PCE (c) vs. composition for the TQm6:TQm12:PC ${ }_{71}$ BM ternary OPV system. 

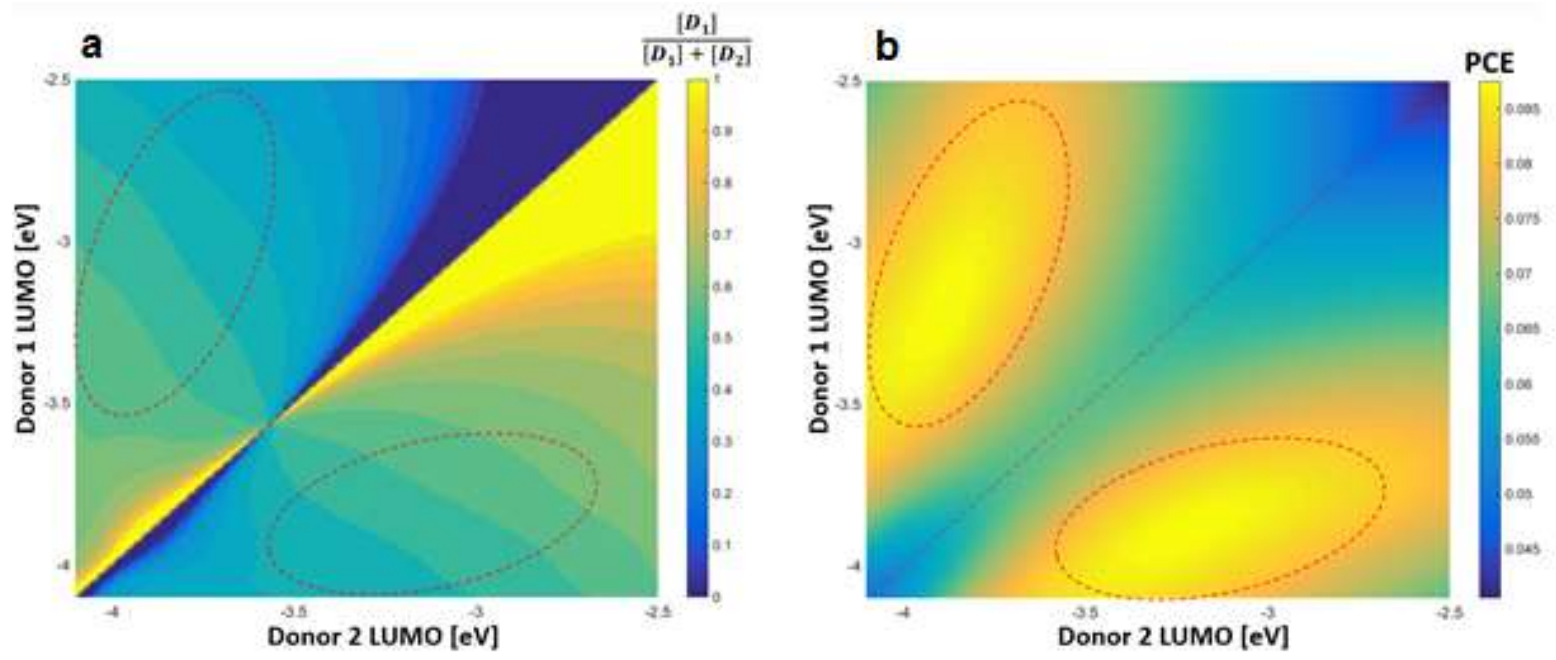

Fig. 5 (a) Optimal fraction of Donor 1 and (b) associated PCE for all the different LUMO level combinations; the HOMO level is fixed at $-5.4 \mathrm{eV}$. The diagonal dashed line indicates equivalent D1 and D2, i.e. a binary system; as expected graphs a and b are anti-symmetric resp. symmetric w.r.t. to this line. The highest overall PCE of $8.75 \%$ is obtained for ternary blends indicated by the dashed ellipses.
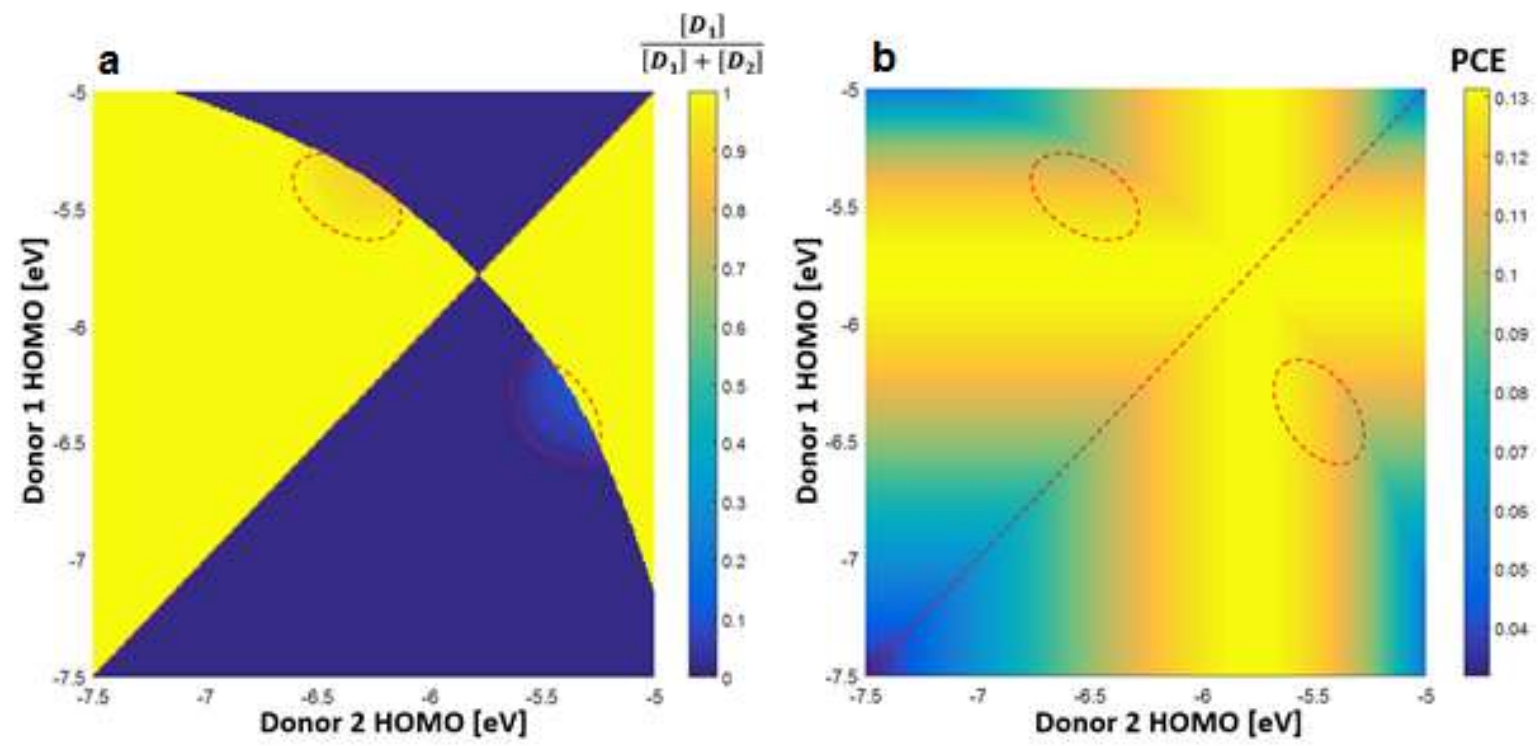

Fig. 6 (a) Optimal fraction of Donor 1 and (b) associated PCE for all different HOMO level combinations for an absorption width FWHM = $0.8 \mathrm{eV}$; the LUMO level is fixed at $-3.8 \mathrm{eV}$; a constant fill factor of 0.65 was used. The diagonal dashed line indicates equivalent D1 and D2, i.e. a binary system. Small ternary regions are indicated by dashed ellipses. 


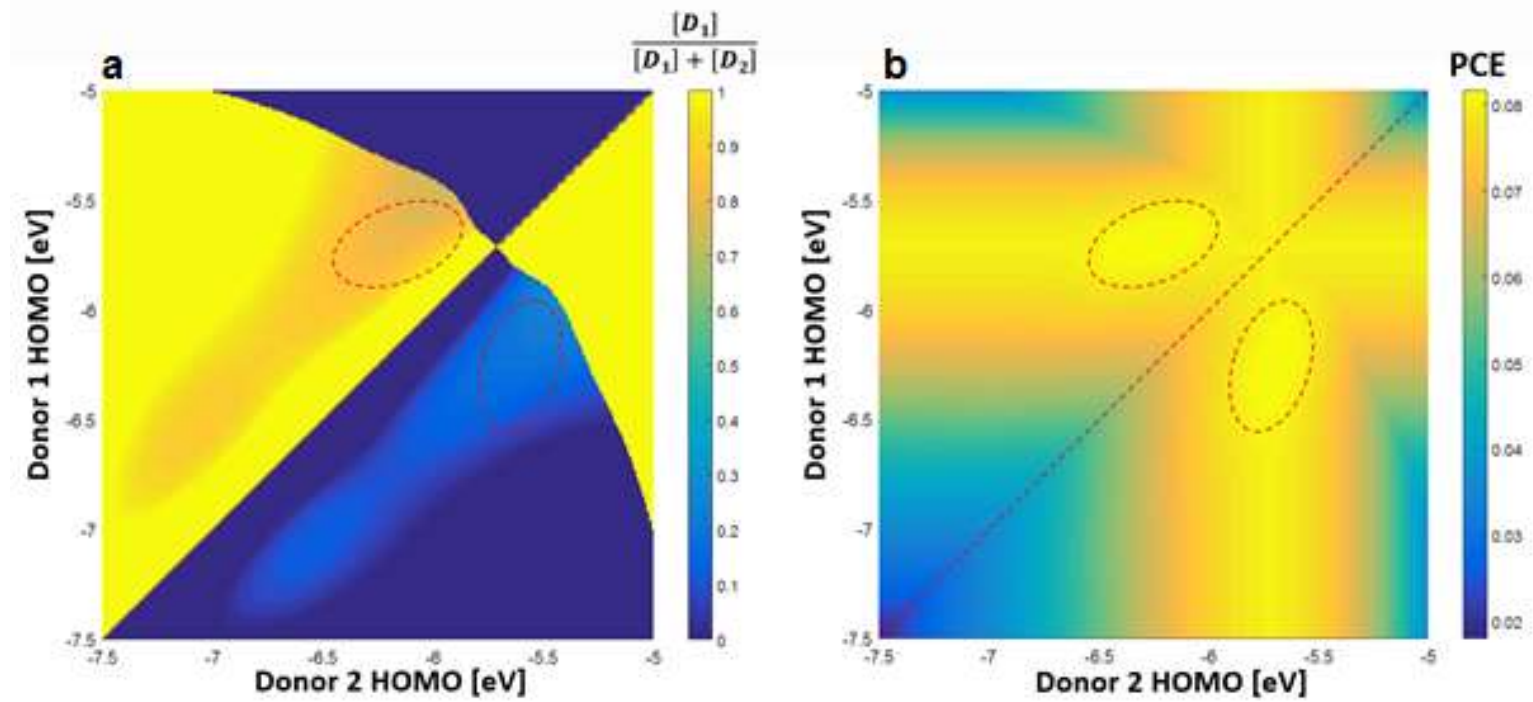

Fig. 7 (a) Optimal fraction of Donor 1 and (b) associated PCE for all different HOMO level combinations for a narrower absorption width (FWHM $=0.5 \mathrm{eV}$ ); the LUMO level is fixed at $3.8 \mathrm{eV}$; a constant fill factor of 0.65 was used. The diagonal dashed line indicates equivalent D1 and D2, i.e. a binary system. Ternary regions indicated by dashed ellipses give an overall optimal PCE of $\sim 8.2 \%$.
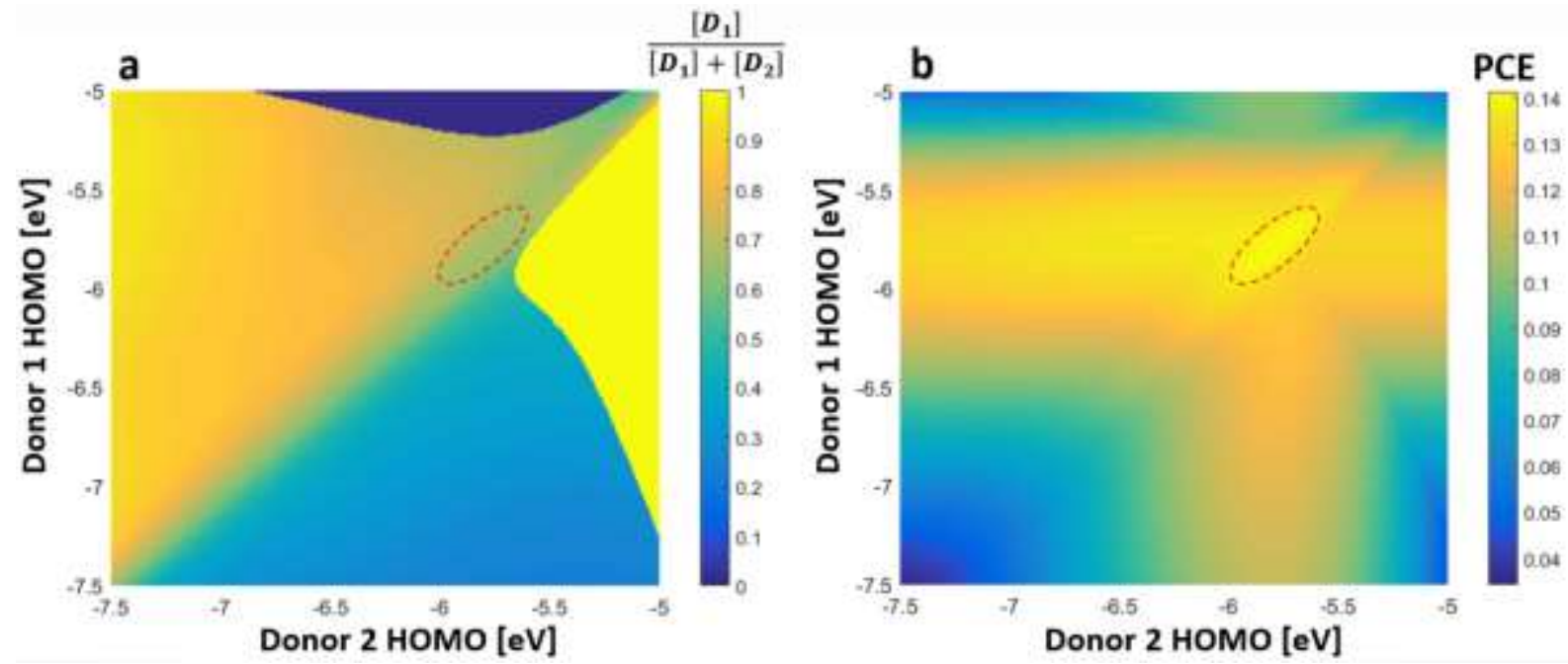

Fig. 8 (a) Optimal fraction of Donor 1 and (b) associated PCE for all different HOMO level combinations. Same calculation parameters as Figure 6 but with a composition-dependent fill factor given by Eq. (13) with $F F_{D 1}=0.65 F F_{D 2}=0.5$ and $a=-$ 0.45 , corresponding to a maximum FF of 0.7 at $f_{D 1}=0.66$. The dashed ellipse indicates the best overall PCE of $\sim 14.1 \%$ at a D1 fraction of 0.66 . 


\section{Supporting Information to}

\section{Open circuit voltage and efficiency in ternary organic photovoltaic blends}

Nikolaos Felekidis ${ }^{1}$, Ergang Wang ${ }^{2}$, Martijn Kemerink ${ }^{1,3}$

${ }^{1}$ Complex Materials and Devices, Department of Physics, Chemistry and Biology (IFM), Linköping University, SE-581 83 Linköping, Sweden

${ }^{2}$ Department of Chemistry and Chemical Engineering, Chalmers University of Technology, SE-412 96 Göteborg, Sweden

${ }^{3}$ Department of Applied Physics, Eindhoven University of Technology, PO Box 513, NL-5600 MB Eindhoven, The Netherlands

E-mail: martijn.kemerink@liu.se

\section{Contents}

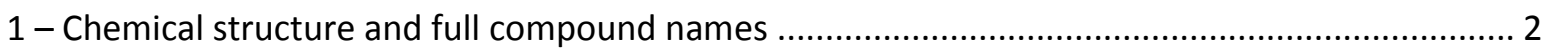

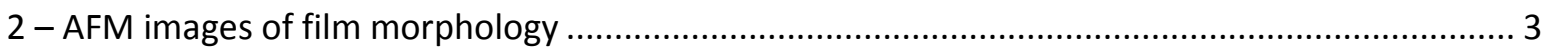

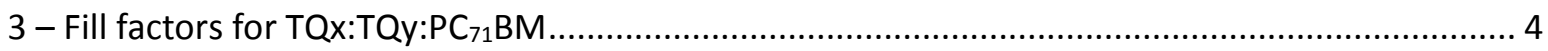

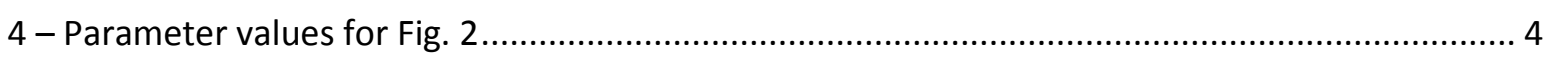

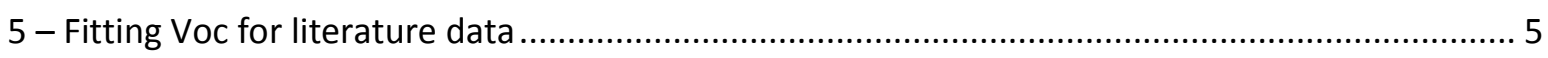

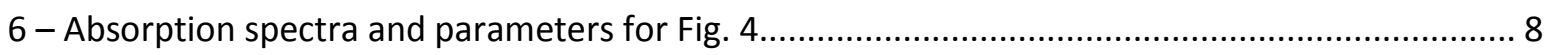

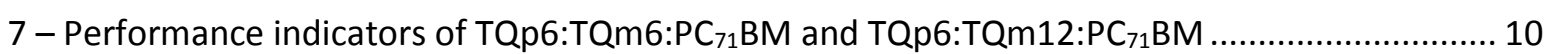

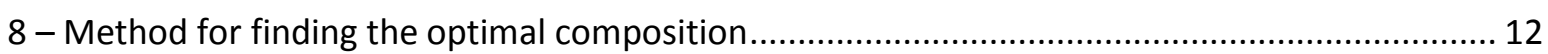

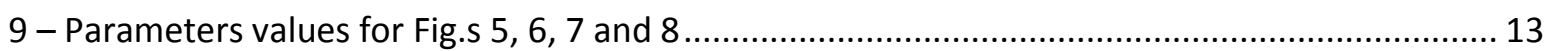

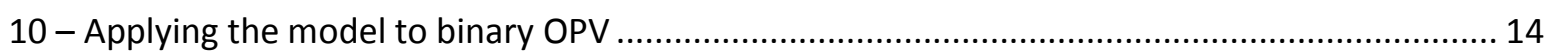

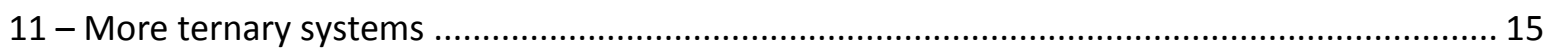

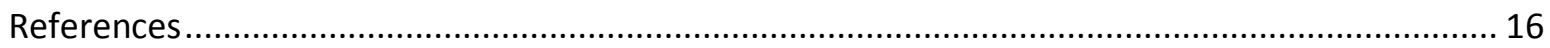




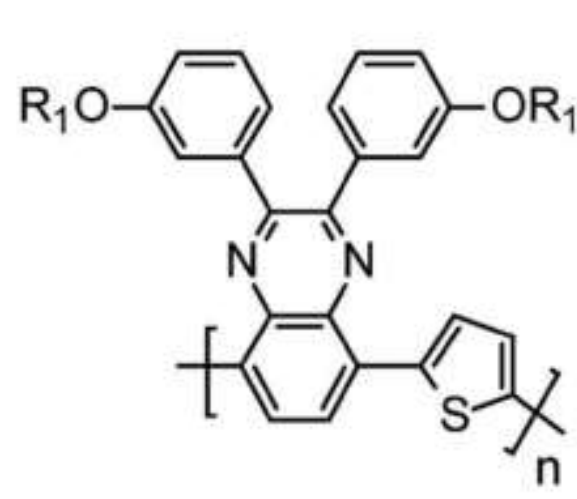

$\mathrm{TQ}_{\mathrm{m6}} \quad \mathrm{R}_{1}=\mathrm{n}-\mathrm{C}_{6} \mathrm{H}_{13}$

$T Q_{m 12} R_{1}=n-C_{12} H_{25}$

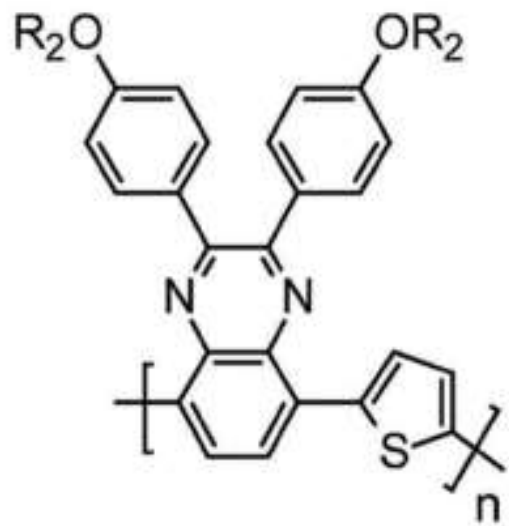

$\mathrm{TQ}_{\mathrm{p6}} \quad \mathrm{R}_{2}=\mathrm{n}-\mathrm{C}_{6} \mathrm{H}_{13}$

Fig. S1. Molecular structure of TQm6,TQm12 and TQp6.

Poly[2,3-bis-(3-hexyloxyphenyl)quinoxaline-5,8-diyl-alt-thiophene-2,5-diyl] (TQm6)

Poly[2,3-bis-(3-dodecyloxyphenyl)quinoxaline-5,8-diyl-alt-thiophene-2,5-diyl] (TQm12)

Poly[2,3-bis-(4-hexyloxyphenyl)quinoxaline-5,8-diyl-alt-thiophene-2,5-diyl] (TQp6) 

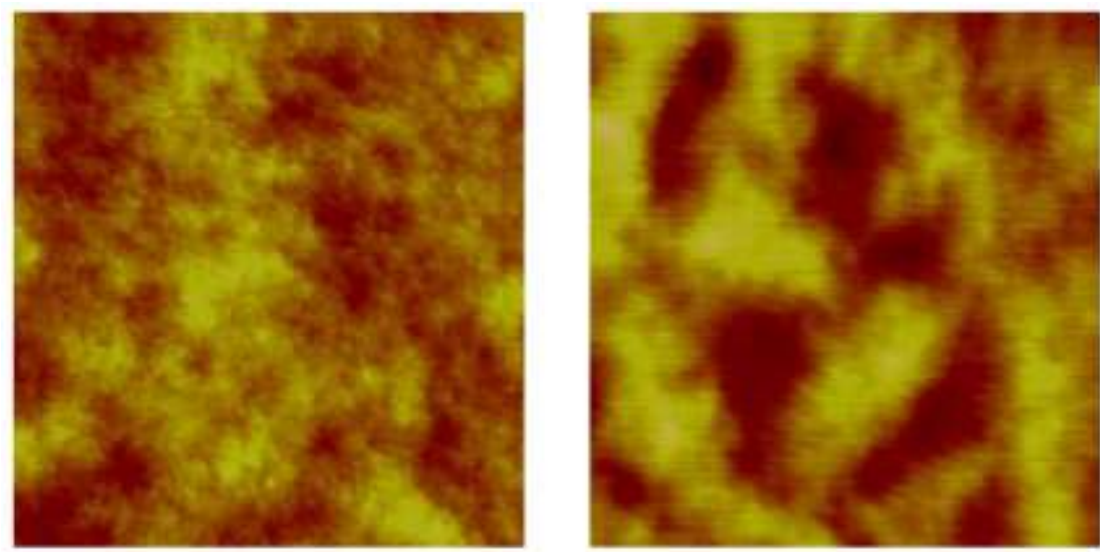

Fig. S2. AFM images of TQm6:TQm12:PC ${ }_{71} \mathrm{BM}$ BHJ OPV. The left (right) picture corresponds to TQm6/(TQm6+TQm12) $=0.5(0.6)$. Scan size is $3 \times 3 \mu \mathrm{m}$, total vertical scale is $10 \mathrm{~nm}$.
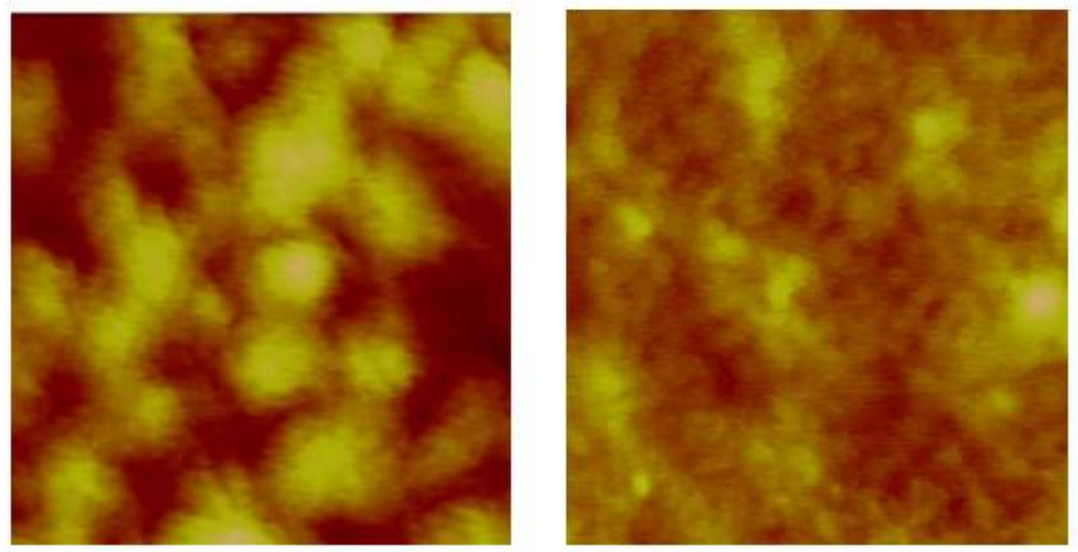

Fig. S3. AFM images of TQp6:TQm6:PC $\mathrm{C}_{71} \mathrm{BM} \mathrm{BHJ} \mathrm{OPV}$. The left (right) picture corresponds to TQp6/TQp6+TQm6 $=0.2(0.6)$. Scan size is $3 \times 3 \mu \mathrm{m}$, total vertical scale is $10 \mathrm{~nm}$. 


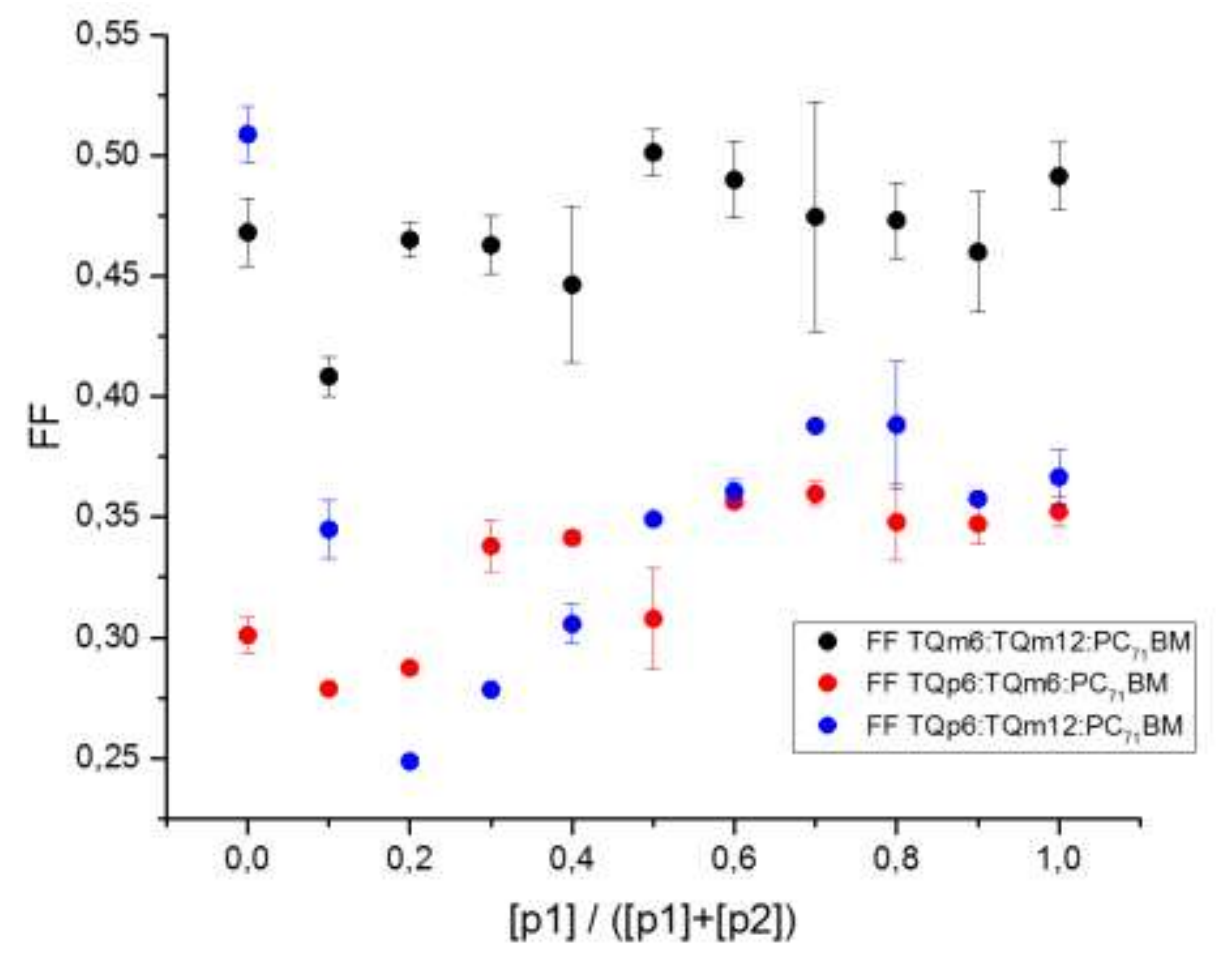

Fig. S4. Fill factors for all three TQx:TQy:PC ${ }_{71} \mathrm{BM}$ polymer combinations.

\section{4 - Parameter values for Fig. 4}

\begin{tabular}{|c|c|c|c|}
\hline$\sigma_{\text {TQm6 }}[\mathrm{eV}]$ & 0.1 & $\sigma_{\text {TQp6 }}[\mathrm{eV}]$ & 0.12 \\
\hline$\sigma_{\text {TQm12 }}[\mathrm{eV}]$ & 0.104 & $\sigma_{\text {TQm12 }}[\mathrm{eV}]$ & 0.104 \\
\hline$\sigma_{\text {РС71BM }}[\mathrm{eV}]$ & 0.12 & $\sigma_{\text {РC71BM }}[\mathrm{eV}]$ & 0.12 \\
\hline occupation & $1.05 \cdot 10^{-3}$ & occupation & $10^{-3}$ \\
\hline DOS & $10^{26}$ & DOS & $10^{26}$ \\
\hline HOMO TQm6 $[\mathrm{eV}]$ & -5.72 & HOMO тQр6 $[\mathrm{eV}]$ & -5.55 \\
\hline HOMO TQm12 $[\mathrm{eV}]$ & -5.76 & HOMO TQm12 $[\mathrm{eV}]$ & -5.76 \\
\hline LUMO Рс71вм $[\mathrm{eV}]$ & -4.1 & LUMO Рс71вм [eV] & -4.1 \\
\hline
\end{tabular}

Table S1. Parameter values used in the simulation model for the fitting of TQm6:TQm12:PC $\mathrm{C}_{71} \mathrm{BM}$ and TQm6:TQm12:PC ${ }_{71} \mathrm{BM}$ assuming constant occupation. 


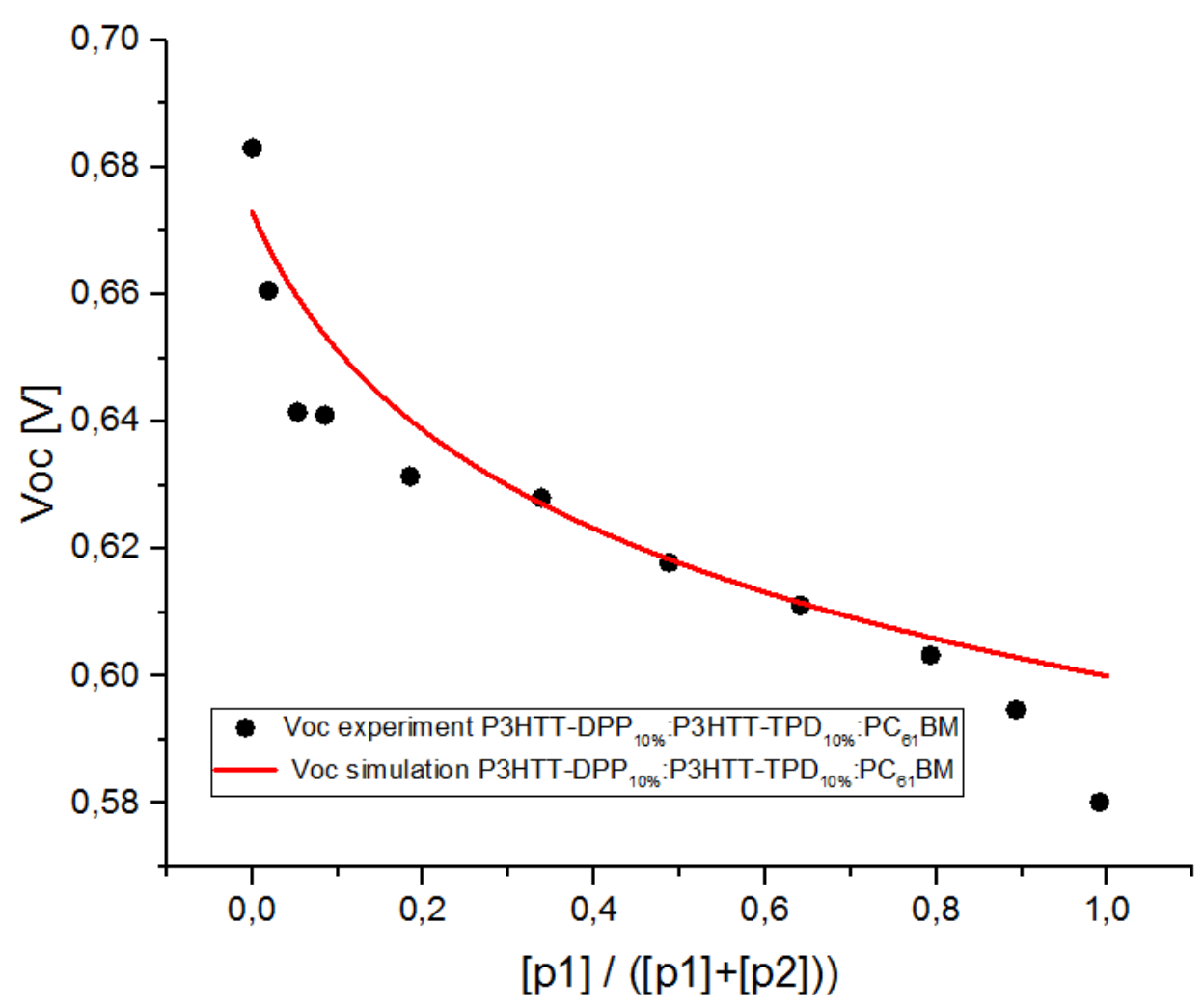

Fig. S5. Fitting of the P3HTT-DPP10\%:P3HTT-TPD10\%:PC61BM ternary BHJ OPV from Khlyabich et al. ${ }^{[1]}$ assuming constant occupation.

\begin{tabular}{|ll|l|}
\hline$\sigma$ P3HTT-DPP10\% & {$[\mathrm{eV}]$} & 0.075 \\
\hline$\sigma$ P3HTT-TPD10\% $\quad[\mathrm{eV}]$ & 0.105 \\
\hline$\sigma$ PC61BM $\quad[\mathrm{eV}]$ & 0.075 \\
\hline OcCupation & $1.2 \cdot 10^{-3}$ \\
\hline total_Po_No & $10^{26}$ \\
\hline HOMO P3HTT-DPP10\% [eV] & -5.25 \\
\hline HOMO P3HTT-TPD10\% [eV] & -5.40 \\
\hline LUMO PC61BM [eV] & -4.10 \\
\hline
\end{tabular}

Table S2. Parameter values used in the simulation model for the fitting of the P3HTT-DPP10\%:P3HTTTPD10\%:PC61BM ternary BHJ OPV from Khlyabich et al. ${ }^{[1]}$ assuming constant occupation. 


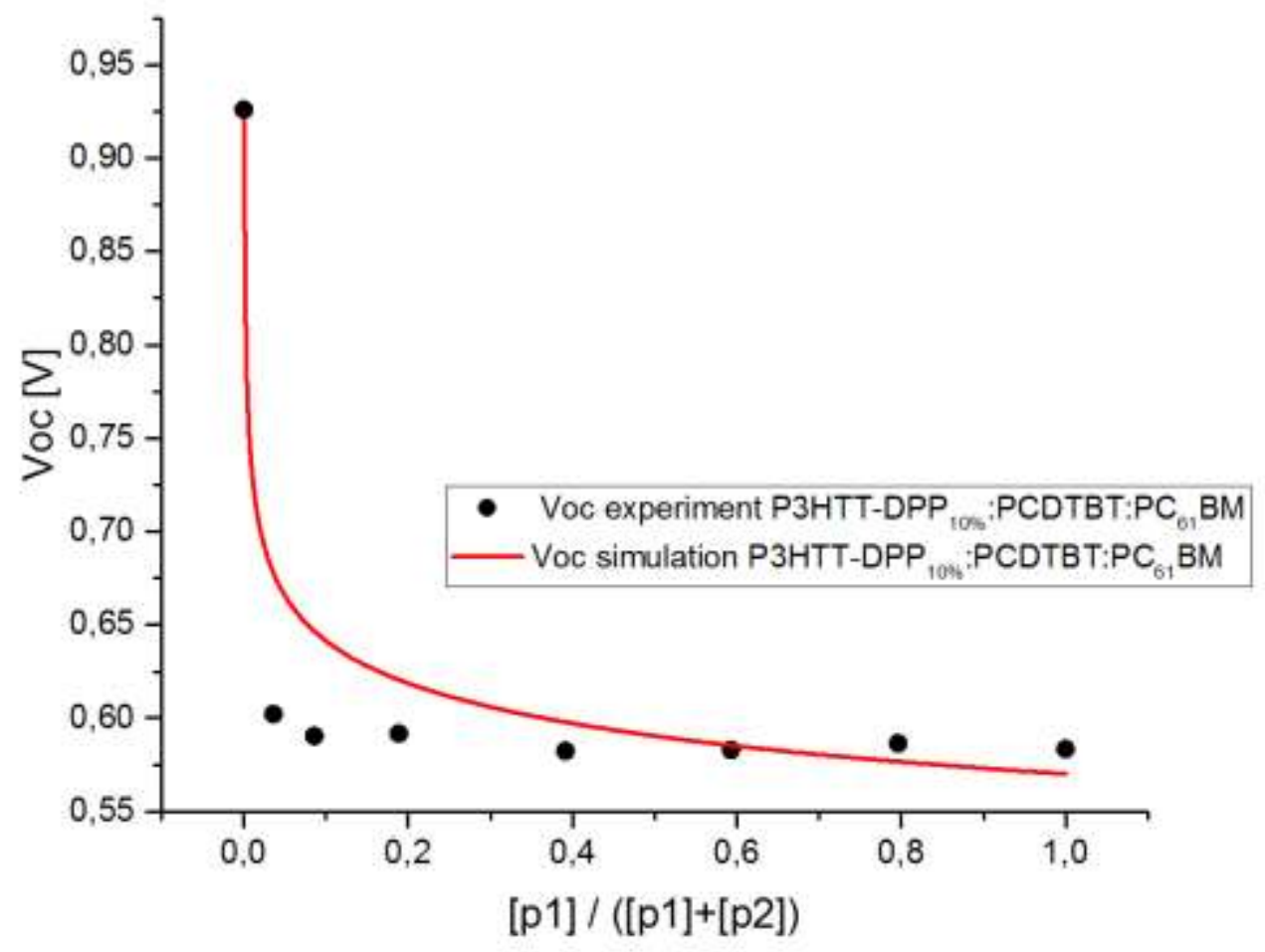

Fig. S6. Fitting of the P3HTT-DPP10\%:PCDTBT:PC61BM ternary BHJ OPV from Khlyabich et al. ${ }^{[1]}$ assuming constant occupation.

\begin{tabular}{|ll|l|}
\hline$\sigma$ P3HTT-DPP10\% $\quad[\mathrm{eV}]$ & 0.075 \\
\hline $\boldsymbol{\sigma}$ P3HTT-PCDTBT $\quad[\mathrm{eV}]$ & 0.075 \\
\hline $\boldsymbol{\sigma}$ PC61BM $\quad[\mathrm{eV}]$ & 0.075 \\
\hline occupation & $7.2 \cdot 10^{-4}$ \\
\hline total_Po_No & $10^{26}$ \\
\hline HOMO P3HTT-DPP10\% [eV] & -5.25 \\
\hline HOMO PCDTBT [eV] & -5.6 \\
\hline LUMO PC61BM [eV] & -4.10 \\
\hline
\end{tabular}

Table S3. Parameter values used in the simulation model for the fitting of the P3HTTDPP10\%:PCDTBT:PC61BM ternary BHJ OPV from Khlyabich et al. ${ }^{[1]}$ assuming constant occupation. 


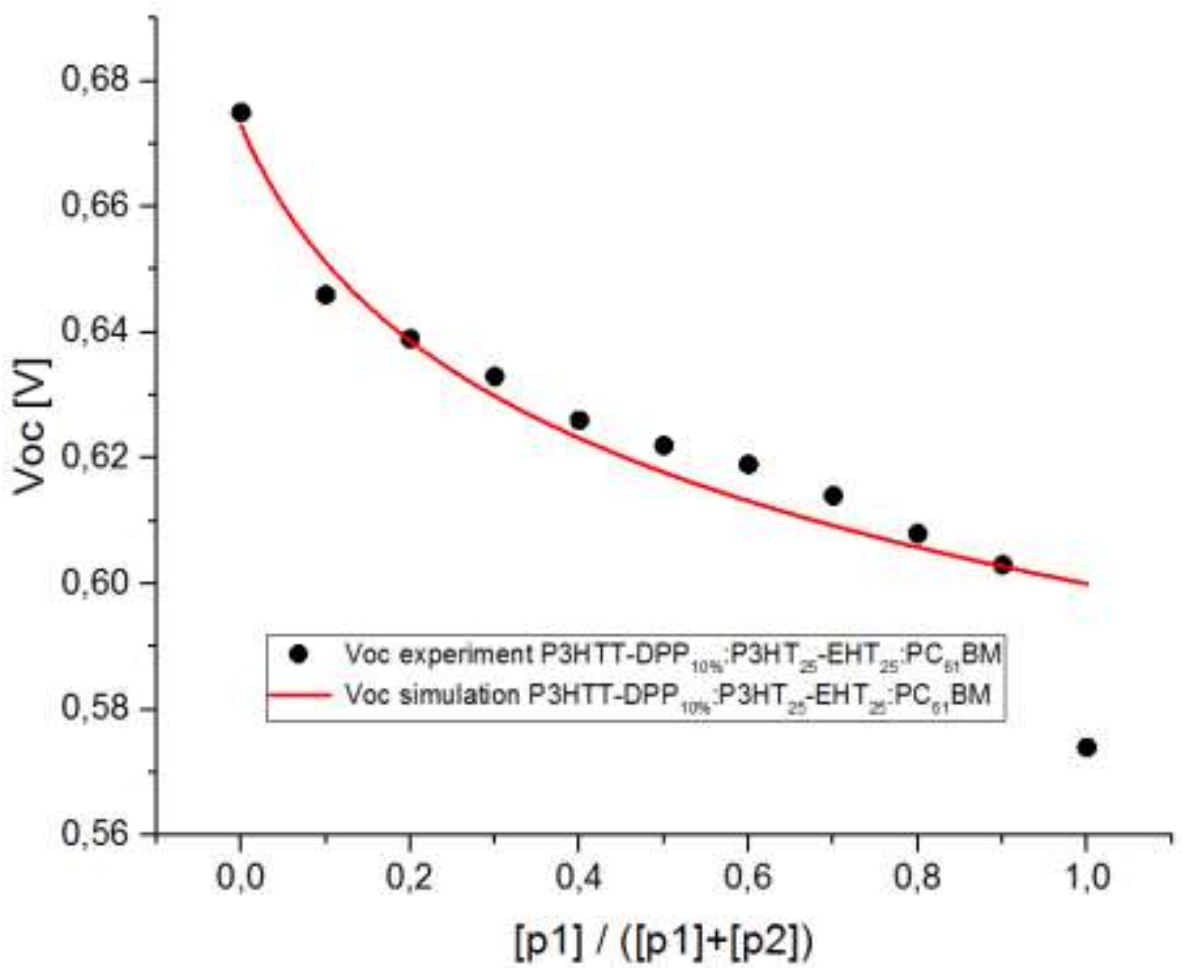

Fig. S7. Fitting of the P3HTT-DPP10\%:P3HT25-EHT25:PC61BM ternary BHJ OPV from Khlyabich et al. ${ }^{[2]}$ assuming constant occupation.

\begin{tabular}{|l|l|}
\hline$\sigma$ P3HTT-DPP10\% $\quad[\mathrm{eV}]$ & 0.075 \\
\hline $\boldsymbol{\sigma}$ P3HT25-EHT25 [eV] & 0.105 \\
\hline $\boldsymbol{\sigma}$ PC61BM $\quad[\mathrm{eV}]$ & 0.075 \\
\hline OcCupation & $1.2 \cdot 10^{-3}$ \\
\hline total_Po_No & $10^{26}$ \\
\hline HOMO P3HTT-DPP10\% [eV] & -5.25 \\
\hline HOMO P3HT25-EHT25 [eV] & -5.40 \\
\hline LUMO PC61BM [eV] & -4.10 \\
\hline
\end{tabular}

Table S4. Parameter values used in the simulation model for the fitting of P3HTT-DPP10\%:P3HT25EHT25:PC61BM from Khlyabich et al. ${ }^{[2]}$ assuming constant occupation. 
6 - Absorption spectra and parameters for Fig. 4

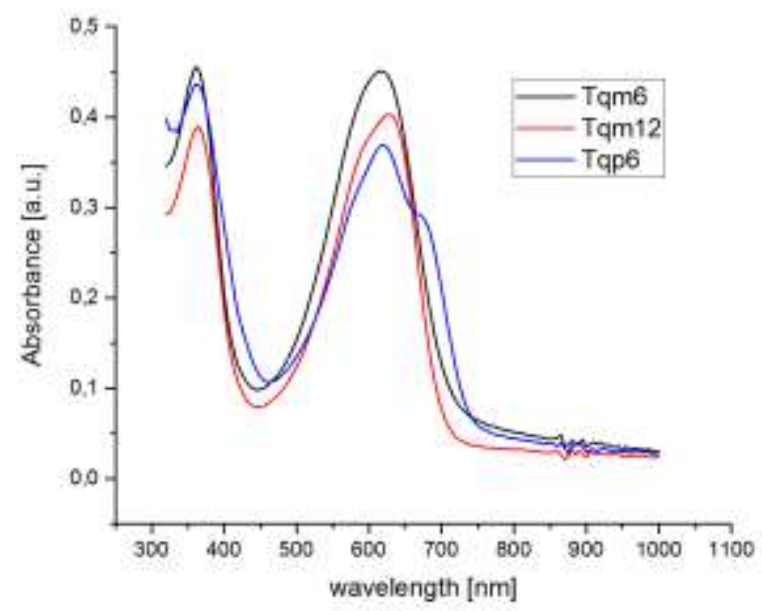

Fig. S8. UV-vis spectra of TQm6, TQm12 and TQp6 polymers. The FWHM were measured to be $148 \mathrm{~nm}$, $138 \mathrm{~nm}$ and $175 \mathrm{~nm}$ for TQm6, TQm12 and TQp6, respectively. Used films are $\sim 80 \mathrm{~nm}$ thick, spin-coated on glass substrates. 


\begin{tabular}{|c|c|}
\hline $\boldsymbol{\sigma}_{\text {TQm6 }}[\mathrm{eV}]$ & 0.1 \\
\hline $\boldsymbol{\sigma}_{\text {TQm12 }}[\mathrm{eV}]$ & 0.09 \\
\hline$\sigma_{\text {рс71вM }[\mathrm{eV}]}$ & 0.12 \\
\hline DOS & $5.5 \cdot 10^{26}$ \\
\hline LUMO TQm6 $_{\text {TQV }}[\mathrm{eV}$ & -3.80 \\
\hline HOMO $_{\text {TQm6 }}[\mathrm{eV}]$ & -5.72 \\
\hline LUMO $_{\text {TQm12 }}[\mathrm{eV}]$ & -3.45 \\
\hline HOMO $_{\text {TQm12 }}[\mathrm{eV}]$ & -5.76 \\
\hline LUMO РC71BM $[\mathrm{eV}]$ & -4.10 \\
\hline IQE & 0.85 \\
\hline lifetime $_{\text {holes }}[\mathrm{s}]$ & $10^{-5}$ \\
\hline lifetime $_{\text {electrons }}[\mathrm{s}]$ & $10^{-5}$ \\
\hline FF & 0.45 \\
\hline FWHM TQm6 $_{\text {Th] }}$ & $148 \cdot 10^{-9}$ \\
\hline FWHM TQm12 $_{\text {Th] }}$ & $138 \cdot 10^{-9}$ \\
\hline Length Device $[\mathrm{m}]$ & $100 \cdot 10^{-9}$ \\
\hline Abs. length Tam6 $[\mathrm{m}]$ & $60 \cdot 10^{-9}$ \\
\hline Abs. length TQm12 $[\mathrm{m}]$ & $95 \cdot 10^{-9}$ \\
\hline
\end{tabular}

Table S5. Parameter values used in the simulation model for the fitting of the experimental Voc, Jsc and PCE data for TQm6:TQm12:PC ${ }_{71} \mathrm{BM}$ in Fig. 4. The length ${ }_{\text {device }}$ corresponds to the mean thickness of the investigated device. As these devices are semi-transparent the device thickness corresponds to the optical path length. 

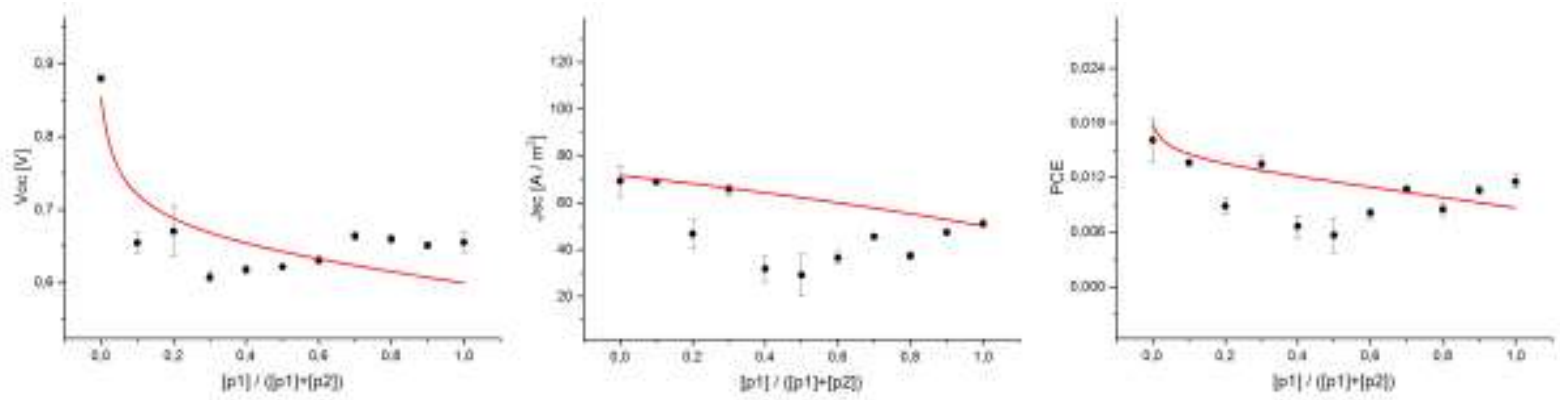

Fig. S9. Simulation of experimental Voc, Jsc, PCE data vs composition for the TQp6:TQm6:PC ${ }_{71} B M$ ternary OPVs. Symbols and lines are experiments and simulations, respectively.

\begin{tabular}{|c|c|}
\hline $\begin{array}{ll}\sigma_{\text {TQp6 }} & {[\mathrm{eV}]}\end{array}$ & 0.12 \\
\hline$\sigma_{\text {TQm6 } 6}[\mathrm{eV}]$ & 0.1 \\
\hline$\sigma_{\text {рс71вм }[\mathrm{eV}]}$ & 0.12 \\
\hline DOS & $10^{26}$ \\
\hline LUMO TQp6 $_{\text {TeV }]}$ & -3.1 \\
\hline HOMO $_{\text {тQp6 }}[\mathrm{eV}]$ & -5.55 \\
\hline LUMO TQm6 $_{\text {таV }}[\mathrm{eV}]$ & -3.3 \\
\hline HOMO $_{\text {TQm6 }}[\mathrm{eV}]$ & -5.72 \\
\hline LUMO PC71BM $[\mathrm{eV}]$ & -4.1 \\
\hline IQE & 0.85 \\
\hline lifetime $_{\text {holes }}[\mathrm{s}]$ & $10^{-5}$ \\
\hline lifetime $_{\text {electrons }}[\mathrm{s}]$ & $10^{-5}$ \\
\hline FF & 0.3 \\
\hline $\begin{array}{ll}\text { FWHM тQp6 } & {[\mathrm{m}]}\end{array}$ & $175 \cdot 10^{-9}$ \\
\hline $\begin{array}{ll}\text { FWHM } \\
\text { тQm6 }\end{array}$ & $148 \cdot 10^{-9}$ \\
\hline Length Device $[\mathrm{m}]$ & $80 \cdot 10^{-9}$ \\
\hline Abs. length тар6 $[\mathrm{m}]$ & $120 \cdot 10^{-9}$ \\
\hline Abs. length тQm6 $[\mathrm{m}]$ & $60 \cdot 10^{-9}$ \\
\hline
\end{tabular}

Table S6. Parameter values used in the simulation model for the fitting of the experimental Voc, $j_{S C}$

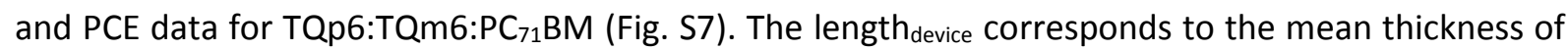
the investigated device. As these devices are semi-transparent the device thickness corresponds to the optical path length. 

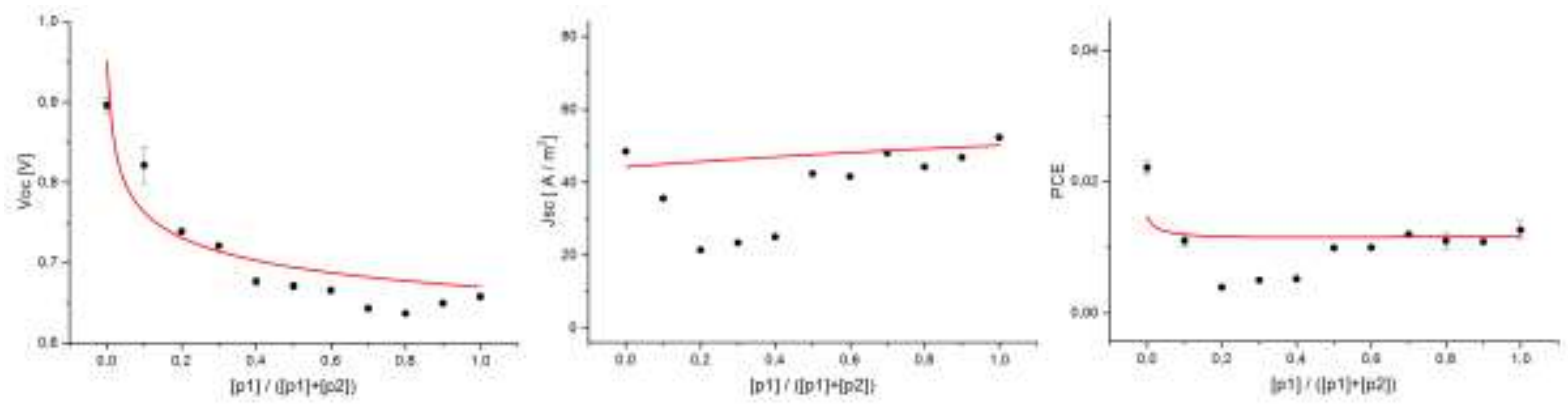

Fig. S10. Simulation of experimental Voc, $j_{S C}$, PCE data vs composition for the TQp6:TQm12:PC ${ }_{71} \mathrm{BM}$ ternary OPVs. Symbols and lines are experiments and simulations, respectively.

\begin{tabular}{|c|c|}
\hline $\begin{array}{ll}\sigma_{\text {TQp6 }} & {[\mathrm{eV}]}\end{array}$ & 0.12 \\
\hline$\sigma_{\text {TQm12 }}[\mathrm{eV}]$ & 0.09 \\
\hline$\sigma_{\text {рс71вм }[\mathrm{eV}]}$ & 0.12 \\
\hline DOS & $3 \cdot 10^{25}$ \\
\hline LUMO TQp6 $_{\text {TUV }}[\mathrm{eV}$ & -3.1 \\
\hline HOMO $_{\text {тQp6 }}[\mathrm{eV}]$ & -5.55 \\
\hline LUMO $_{\text {TQm12 }}[\mathrm{eV}]$ & -3.15 \\
\hline HOMO $_{\text {TQm12 }}[\mathrm{eV}]$ & -5.76 \\
\hline LUMO PC71BM $[\mathrm{eV}]$ & -4.1 \\
\hline IQE & 0.85 \\
\hline lifetime $_{\text {holes }}[\mathrm{s}]$ & $10^{-5}$ \\
\hline lifetime $_{\text {electrons }}[\mathrm{s}]$ & $10^{-5}$ \\
\hline $\mathbf{F F}$ & 0.35 \\
\hline FWHM тQp6 $[\mathrm{m}]$ & $175 \cdot 10^{-9}$ \\
\hline $\begin{array}{ll}\text { FWHM TQm12 } & {[\mathrm{m}]}\end{array}$ & $138 \cdot 10^{-9}$ \\
\hline Length $_{\text {Device }}[\mathrm{m}]$ & $80 \cdot 10^{-9}$ \\
\hline Abs. length тQp6 $[\mathrm{m}]$ & $120 \cdot 10^{-9}$ \\
\hline Abs. length тQm12 [m] & $95 \cdot 10^{-9}$ \\
\hline
\end{tabular}

Table S7. Parameter values used in the simulation model for the fitting of the experimental Voc, Jsc and PCE data for TQp6:TQm12:PC ${ }_{71} \mathrm{BM}$ (Fig. S8). The length ${ }_{\text {device }}$ corresponds to the mean thickness of the investigated device. As these devices are semi-transparent the device thickness corresponds to the optical path length. 

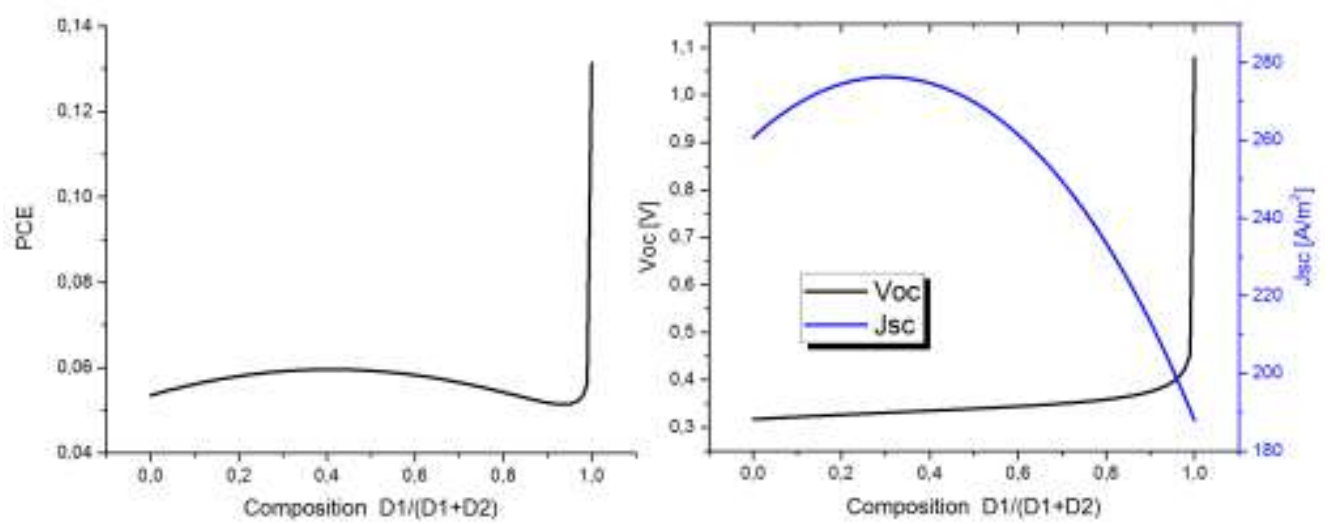

Fig. S11. (left) PCE vs composition plot for the D1, D2 pair that gives the highest overall PCE in Fig. 6. The optimal composition that gives the best PCE is in this case $100 \%$ of $D 1, D 1 /(D 1+D 2)=1$, i.e. a pure binary system. The sudden jumps in optimal composition observed in Figs. 6, 7 that do not sit at the binary line $(y=x)$ are associated with the presence of two local maxima in this curve. At the discontinuity the dominant maximum changes from ternary to binary or vice versa. (right) Corresponding Voc and $j_{S C}$. 


\begin{tabular}{|c|c|}
\hline$\sigma_{\text {Donor1 }}[\mathrm{eV}]$ & 0.075 \\
\hline$\sigma_{\text {Donor2 }}[\mathrm{eV}]$ & 0.075 \\
\hline$\sigma_{\text {Acceptor }}[\mathrm{eV}]$ & 0.075 \\
\hline DOS & $10^{26}$ \\
\hline HOMO $_{\text {Donor1 }}[\mathrm{eV}]$ & -5.4 \\
\hline LUMO Donor1 $[\mathrm{eV}]$ & $-2.5 \ldots-4.1$ \\
\hline HOMO Donor2 $[\mathrm{eV}]$ & -5.4 \\
\hline LUMO Donor2 $[\mathrm{eV}]$ & $-2.5 \ldots-4.1$ \\
\hline LUMO $_{\text {Acceptor }}[\mathrm{eV}]$ & -4.1 \\
\hline IQE & 0.85 \\
\hline lifetime $_{\text {holes }}[\mathrm{s}]$ & $10^{-5}$ \\
\hline lifetime $_{\text {electrons }}[\mathrm{s}]$ & $10^{-5}$ \\
\hline FF & 0.65 \\
\hline FWHM $_{\text {Donor1 }}[\mathrm{m}]$ & $175 \cdot 10^{-9}$ \\
\hline $\begin{array}{ll}\text { FWHM Donor2 } & {[\mathrm{m}]}\end{array}$ & $175 \cdot 10^{-9}$ \\
\hline Length Device $[\mathrm{m}]$ & $240 \cdot 10^{-9}$ \\
\hline Abs. length Donor1 $[\mathrm{m}]$ & $120 \cdot 10^{-9}$ \\
\hline Abs. length ${ }_{\text {Donor2 }}[\mathrm{m}]$ & $120 \cdot 10^{-9}$ \\
\hline
\end{tabular}

Table S8. Parameter values used for the LUMO variation simulations in Fig. 5, inspired by the work of

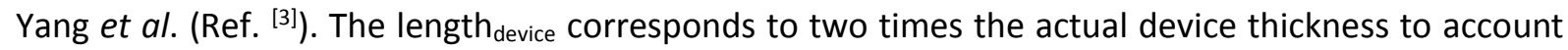
for the fact that the optical path length (in lowest order) is twice the device thickness in devices with a reflective back electrode. For Figs. 6, 7 and 8 the same parameters are used, except (a) the HOMO levels that are varied, fixing the (common) LUMO level at $-3.8 \mathrm{eV}$; (b) the absorption spectra are calculated on an energy axis, using FWHM $=0.80 \mathrm{eV}$ (for Fig. 6) or FWHM $=0.50 \mathrm{eV}$ (for Fig. 7) for both D1 and D2 and (c) for Fig. 8 the fill factor was not constant but given by $F F\left(f_{D 1}\right)=a f_{D 1}^{2}+\left(F F_{D 1}-F F_{D 2}-a\right) f_{D 1}+F F_{D 2}$. 


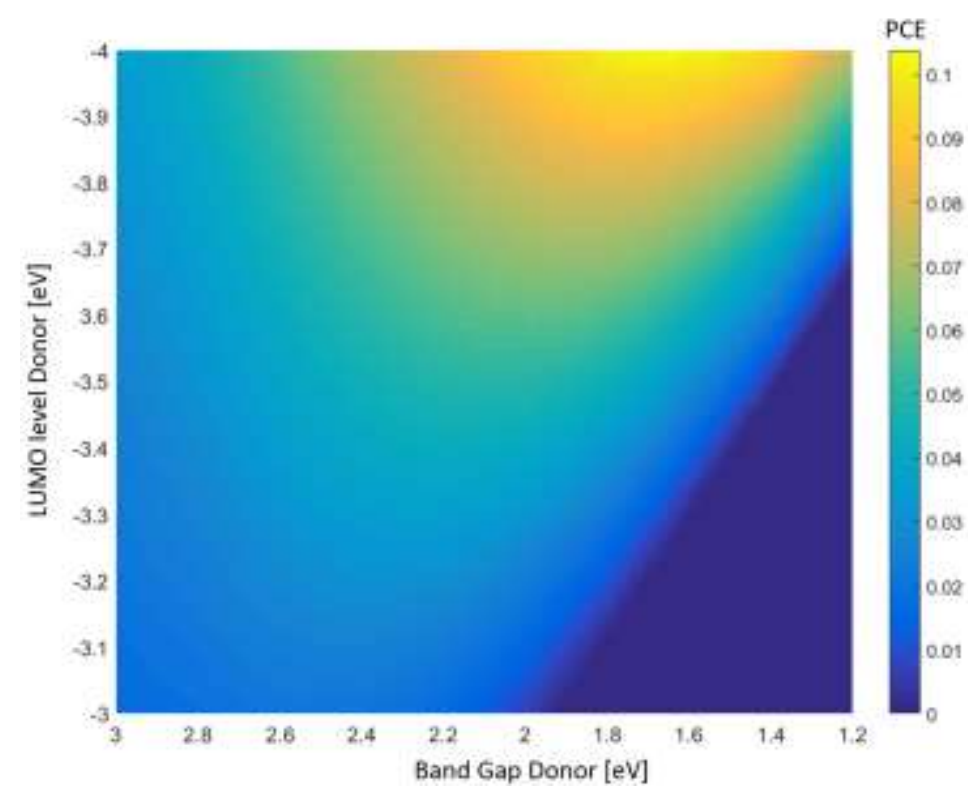

Fig. S12. PCE vs Donor LUMO-HOMO level for binary devices, reproducing the work of Scharber et al. ${ }^{[4]}$

\begin{tabular}{|l|l|}
\hline$\sigma_{\text {Donor } \quad[\mathrm{eV}]}$ & 0.075 \\
\hline $\boldsymbol{\sigma}_{\text {Acceptor }[\mathrm{eV}]}$ & 0.075 \\
\hline DOS & $10^{26}$ \\
\hline Gap Donor $[\mathrm{eV}]$ & $1.2 \ldots 3.0$ \\
\hline LUMO $_{\text {Donor }[\mathrm{eV}]}$ & $-3.0 \ldots-4.0$ \\
\hline LUMO $_{\text {Acceptor }[\mathrm{eV}]}$ & -4.3 \\
\hline IQE & 0.85 \\
\hline lifetime & $10^{-5}$ \\
\hline lifetime \\
\hline electrons [s] $[\mathrm{s}]$ & $10^{-5}$ \\
\hline FF & 0.65 \\
\hline Length Device [m] & $240 \cdot 10^{-9}$ \\
\hline Abs. length Donor1 [m] & $60 \cdot 10^{-9}$ \\
\hline
\end{tabular}

Table 59. Parameter values used for reproducing the work of Scharber et al. ${ }^{[3]}$ Following Ref. ${ }^{[3]}$, the

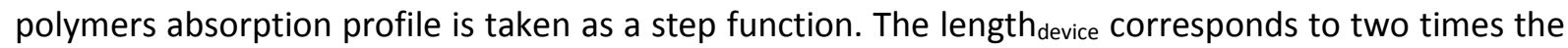
actual device thickness to account for the fact that the optical path length (in lowest order) is twice the device thickness in devices with a reflective back electrode. The IQE value of 0.85 differs from the 0.65 used by Scharber et al. and compensates for the fact that our (more advanced) definition of Voc leads to lower Voc values. 

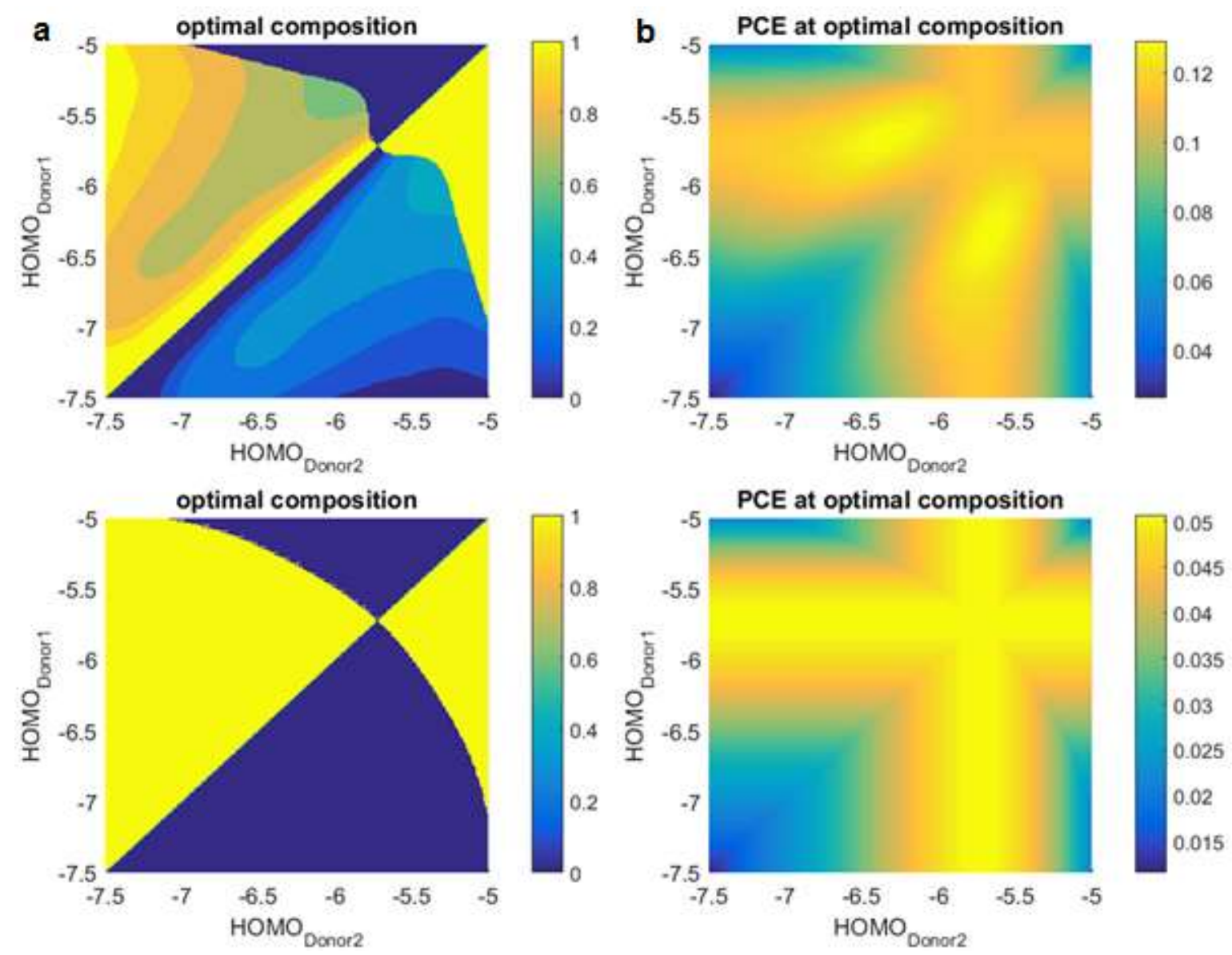

Fig. S13. (a) Optimal fraction of Donor 1 and (b) associated PCE for all different HOMO level combinations for a narrower absorber (FWHM $=0.5 \mathrm{eV}$ ) of high (top, $L_{D}=50 \mathrm{~nm}$ ) and low (bottom, $L_{D}$ $=240 \mathrm{~nm}$ ) absorption strength; the LUMO level is fixed at $-3.8 \mathrm{eV}$, the optical path length is $240 \mathrm{~nm}$. The other parameters are as in Table T7 and Figs. 5,6,7. The images illustrate that narrow but strong absorbers give rise to systems where ternary compositions offer improved performance over binary ones whereas a weaker absorption shifts the balance to binary-dominated ones. 


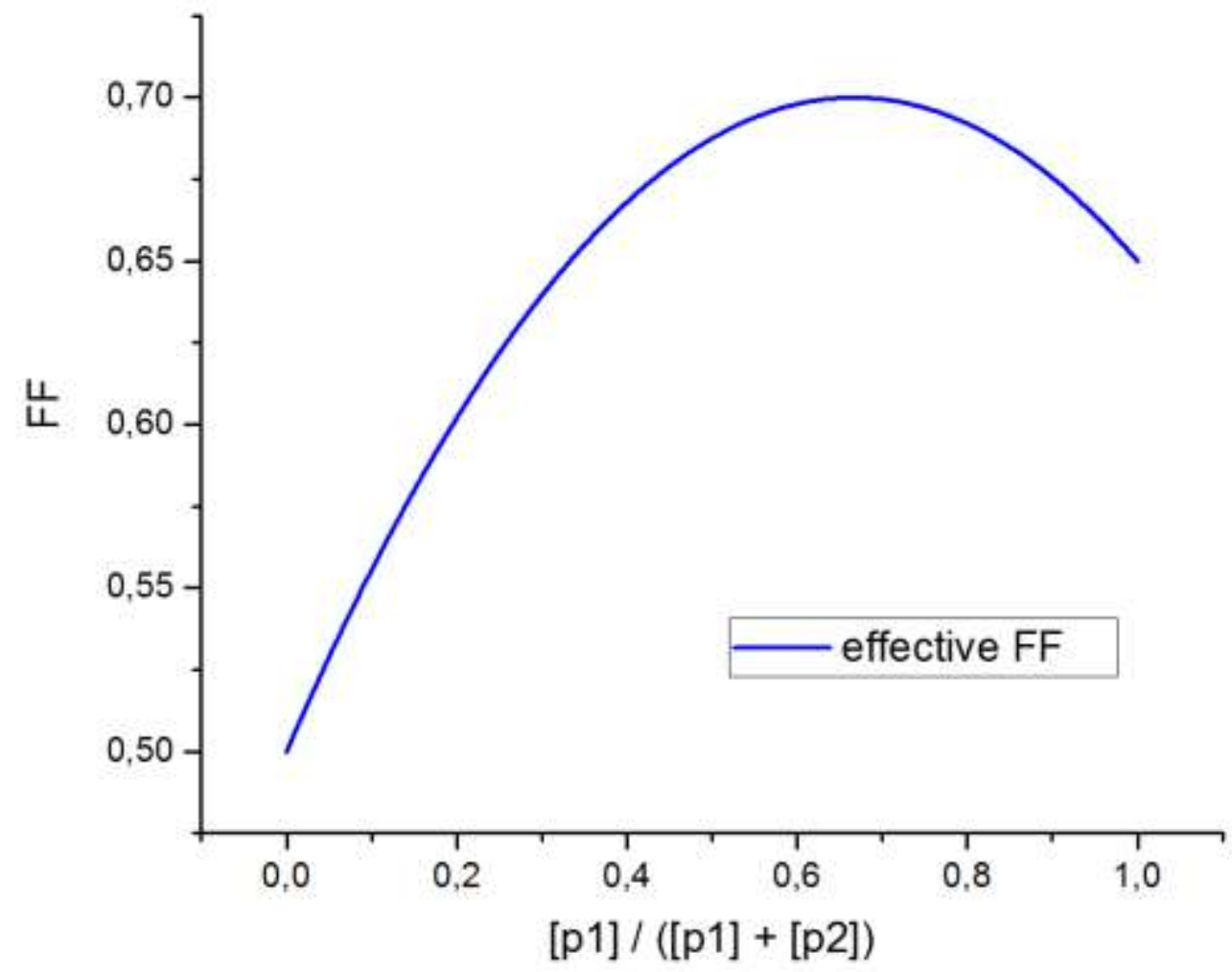

Fig. S14. Effective FF for $\mathrm{FF}_{\mathrm{Donor} 1}=0.65, \mathrm{FF}_{\mathrm{Donor2}}=0.5$ and $\alpha=-0.45$. The $\mathrm{FF}$ is asymmetric with respect to donor composition due to the unequal $\mathrm{FF}$ at the binary extremities and reaches a maximum of 0.70 at $66 \%$ Donor1/ (Donor1+Donor2) composition.

\section{References}

[1] P. P. Khlyabich, Andrey E. Rudenko, Barry C. Thompson, and Yueh-Lin Loo, Adv. Funct. Mater. 2015, 25, 5557-5563

[2] P. P. Khlyabich, B. Burkhart, B. C. Thompson, J. Am. Chem. Soc. 2012, 134, 9074.

[3] Y. (Michael) Yang, W. Chen, L. Dou, W.-H. Chang, H.-S. Duan, B. Bob, G. Li, Y. Yang, Nat. Photonics 2015, advance online publication, DOI 10.1038/nphoton.2015.9.

[4] M. C. Scharber, D. Mühlbacher, M. Koppe, P. Denk, C. Waldauf, A. J. Heeger, C. J. Brabec, Adv. Mater. 2006, 18, 789. 